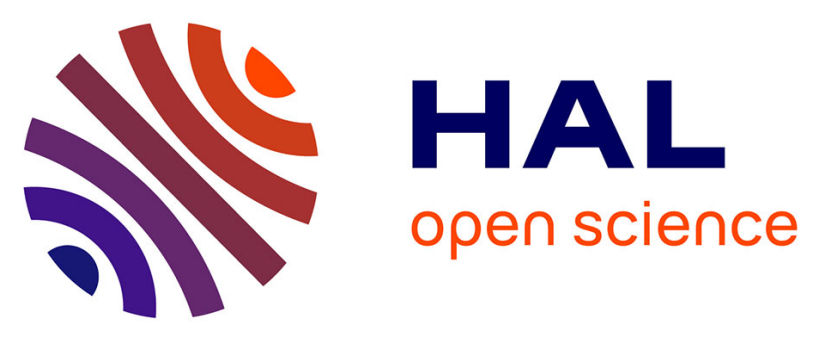

\title{
Adsorption of Organic Dyes on Magnetic Iron Oxide Nanoparticles. Part I: Mechanisms and Adsorption-Induced Nanoparticle Agglomeration
}

Delphine Talbot, Jordy Queiros Campos, Blanca Checa-Fernandez, Jéssica Marins, Claire Lomenech, Charlotte Hurel, Guilhem Godeau, Maxime Raboisson-Michel, Gregory Verger-Dubois, Layaly Obeid, et al.

\section{To cite this version:}

Delphine Talbot, Jordy Queiros Campos, Blanca Checa-Fernandez, Jéssica Marins, Claire Lomenech, et al.. Adsorption of Organic Dyes on Magnetic Iron Oxide Nanoparticles. Part I: Mechanisms and Adsorption-Induced Nanoparticle Agglomeration. ACS Omega, 2021, 6 (29), 10.1021/acsomega.1c02401 . hal-03291969

\section{HAL Id: hal-03291969 \\ https://hal.univ-cotedazur.fr/hal-03291969}

Submitted on 7 Jan 2022

HAL is a multi-disciplinary open access archive for the deposit and dissemination of scientific research documents, whether they are published or not. The documents may come from teaching and research institutions in France or abroad, or from public or private research centers.
L'archive ouverte pluridisciplinaire HAL, est destinée au dépôt et à la diffusion de documents scientifiques de niveau recherche, publiés ou non, émanant des établissements d'enseignement et de recherche français ou étrangers, des laboratoires publics ou privés. 


\section{Adsorption of Organic Dyes on Magnetic Iron Oxide Nanoparticles. Part I: Mechanisms and Adsorption-Induced Nanoparticle Agglomeration}

Delphine Talbot, Jordy Queiros Campos, Blanca L. Checa-Fernandez, Jéssica A. Marins, Claire Lomenech, Charlotte Hurel, Guilhem D. Godeau, Maxime Raboisson-Michel, Gregory Verger-Dubois, Layaly Obeid, Pavel Kuzhir, and Agnès Bee*

Cite This: ACS Omega 2021, 6, 19086-19098

Read Online

ABSTRACT: This series of two papers is devoted to the effect of organic dye (methylene blue, MB; or methyl orange, MO) adsorption on the surface of either bare or citrate-coated magnetic iron oxide nanoparticles (IONPs) on their primary agglomeration (in the absence of an applied magnetic field) and secondary fieldinduced agglomeration. The present paper (Part I) is focused on physicochemical mechanisms of dye adsorption and adsorptioninduced primary agglomeration of IONPs. Dye adsorption to oppositely charged IONPs is found to be mostly promoted by electrostatic interactions and is very sensitive to $\mathrm{pH}$ and ionic

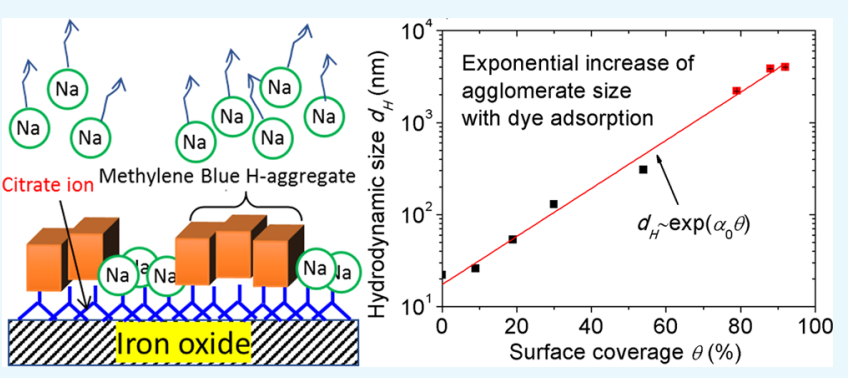
strength variations. The shape of adsorption isotherms is correctly reproduced by the Langmuir law. For the particular MB/citrated IONP pair, the maximum surface density of adsorbed MB seems to correspond to the packing density of an adsorbed monolayer rather than to the surface density of the available adsorption sites. $\mathrm{MB}$ is shown to form $\mathrm{H}$-aggregates on the surface of citrate-coated IONPs. The effective electric charge on the IONP surface remains nearly constant in a broad range of surface coverages by MB due to the combined action of counterion exchange and counterion condensation. Primary agglomeration of IONPs (revealed by an exponential increase of hydrodynamic size with surface coverage by MB) probably comes from correlation attractions or $\pi$-stacking aromatic interactions between adsorbed $\mathrm{MB}$ molecules or $\mathrm{H}$-aggregates. From the application perspective, the maximum adsorption capacity is $139 \pm 4 \mathrm{mg} / \mathrm{g}$ for the MB/citrated IONP pair $(\mathrm{pH}=4-11)$ and $257 \pm 16 \mathrm{mg} / \mathrm{g}$ for the MO/bare IONP pair $(\mathrm{pH} \sim 4)$. Citrated IONPs have shown a good potential for their reusability in water treatment, with the adsorption efficiency remaining about 99\% after nine adsorption/desorption cycles.

\section{INTRODUCTION}

Magnetic manipulation of nanoparticles is a smart tool for various environmental and biomedical applications, such as water remediation from pollutant molecules, ${ }^{1,2}$ high-sensitivity immunoassays, ${ }^{3}$ cancer treatment by controlled drug delivery, hyperthermia or mechanical destruction of cells, ${ }^{4-6}$ protein purification, ${ }^{7}$ gene transfection, ${ }^{8}$ etc. In most of these applications, magnetic nanoparticles bear on their surface adsorbed molecules (either pollutants or biomolecules), which should either be delivered to the target site (drug delivery, gene transfection) or be extracted from the solvent (immunoassays, protein purification, water remediation). Unfortunately, these technics have strong limitations related to low efficiency of the magnetic manipulation of nanoparticles because of their strong Brownian motion and low efficiency of their separation from the suspending fluid (magnetic separation). However, molecules adsorbed on the nanoparticle surface often reduce repulsive colloidal interactions between nanoparticles and could provoke some agglomeration of nanoparticles. Such agglomeration in the absence of an applied magnetic field leads to an increase of the effective size of nanoparticles (or rather primary agglomerates), and once the magnetic field is applied, the magnetic force acting on primary agglomerates is strongly amplified as compared to the situation of single nonaggregated nanoparticles. In this case, the adsorbed molecules not only fulfill their function in water remediation or biomedical applications but also allow a drastic enhancement of nanoparticle manipulation by magnetic fields, thereby broadening the application fields of magnetic nanoparticles.

Despite such an evident technological benefit, the fundamental bases of this phenomenon have still not received

Received: May 7, 2021

Accepted: July 5, 2021

Published: July 19, 2021

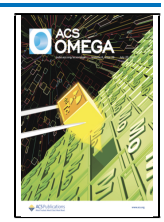


merited attention. The existing literature focuses on the following two distinct aspects of the current problem: (a) nanoparticle agglomeration induced by a change of physicochemical conditions when dispersed in physiological media; $^{9-12}$ and (b) enhancement of the magnetophoretic mobility or increase of the magnetic separation efficiency of magnetic nanoparticles thanks to their field-induced clustering $^{13,14}$ or phase separation. ${ }^{15,16}$ However, the combination of both aforementioned aspects has, to the best of our knowledge, never been studied. Thus, the objective of a series of two present papers (Parts I and II) is to establish the effect of the quantity of adsorbed molecules on the surface of magnetic nanoparticles on the following phenomena: (a) the primary agglomeration occurring due to partial screening of the repulsive interactions between nanoparticles (Part I); (b) the secondary agglomeration induced by dipolar interactions between primary agglomerates in the presence of a magnetic field (Part II); and (c) magnetic separation of nanoparticles under gradient magnetic fields (Part II). To this purpose, we use either bare or anionic (negatively charged) citrate-coated iron oxide nanoparticles (IONP) dispersed in deionized water and either anionic methyl orange (MO) or cationic methylene blue (MB) organic dyes as model pollutants, which adsorb onto the IONP surface and provoke their primary agglomeration, as tested by dynamic light scattering (DLS). This paper (Part I) is focused on adsorption mechanisms of these dyes, and special attention is paid to the citrated MB/IONP pair, for which the primary agglomeration of nanoparticles is analyzed in detail. The considered pair of the oppositely charged MB/ IONP complex should allow discerning some general effects of molecular adsorption on nanoparticle agglomeration valid for other adsorbent/adsorbate pairs, as long as attractive interactions between adsorbent nanoparticles induced by adsorbed molecular layers are known.

Apart from this fundamental aspect, this paper (Part I) has a second objective to evaluate the potential use of IONP for water treatment through adsorption experiments on $\mathrm{MB}$ and $\mathrm{MO}$ dyes. The influence of $\mathrm{pH}$, contact time, and dye concentration on adsorption efficiency is investigated. The reusability of the magnetic composite is assessed through adsorption/desorption cycles. A detailed physicochemical study of our system and the control of the synthesis parameters allowed us to predict the number of active sites involved in the adsorption of the pollutants, which were then compared with experimental adsorption results.

\section{RESULTS AND DISCUSSION}

2.1. Nanoparticle/Ferrofluid Characterization. IONPs were synthetized by coprecipitation of iron salts in an alkaline medium using Massart's method. ${ }^{17,18}$ Bare nanoparticles were dispersed in a dilute aqueous nitric acid solution forming the so-called "acid ferrofluid" (FFA sample). Alternatively, synthesized IONPs were coated by a citrate monolayer and dispersed in a dilute aqueous solution of trisodium citrate at neutral $\mathrm{pH}$ forming the so-called "citrated ferrofluid" (FFCit sample). The IONP synthesis and dispersion protocols are described in detail in Section 4.2.

IONPs were carefully characterized before studying their adsorption properties. The physicochemical parameters of the two ferrofluids are reported in Table 1 , including the molar concentration of iron, $C_{\mathrm{Fe}}(1.70 \mathrm{~mol} / \mathrm{L}$ for FFA and $1.15 \mathrm{~mol} /$ $\mathrm{L}$ for FFCit), as well as the weight concentration $c_{\mathrm{w}}$ and volume fraction $\varphi$ of IONP.
Table 1. Physicochemical Properties of Parent Ferrofluids

\begin{tabular}{lll} 
& \multicolumn{1}{c}{ FFA } & \multicolumn{1}{c}{ FFCit } \\
$C_{\mathrm{Fe}}(\mathrm{mol} / \mathrm{L})$ & $1.70 \pm 0.05$ & $1.15 \pm 0.05$ \\
$c_{\mathrm{w}}(\mathrm{g} / \mathrm{L})$ & $136 \pm 5$ & $92 \pm 5$ \\
$\varphi$ & $2.7 \pm 0.1 \%$ & $1.8 \pm 0.1 \%$ \\
$d_{\mathrm{M}}\left(\sigma_{\mathrm{M}}\right)(\mathrm{nm})$ & $7.8(0.4)$ & $7.6(0.36)$ \\
$M_{\mathrm{s}}(\mathrm{A} / \mathrm{m})$ & $(3.1 \pm 0.1) \times 10^{5}$ & $(3.1 \pm 0.1) \times 10^{5}$ \\
$d_{\mathrm{H}}(\mathrm{PDI})(\mathrm{nm})$ & $21(0.21)$ & $22(0.23)$ \\
$c_{\mathrm{w}}(\mathrm{g} / \mathrm{L})$ of the DLS sample & $\sim 0.2$ & 0.18 \\
$S\left(\mathrm{~m}^{2} / \mathrm{g}\right)$ & $\sim 152$ & $\sim 123-148$ \\
$Q_{\text {tot }}(\mathrm{mmol} / \mathrm{g})$ & $0.39 \pm 0.01$ & $0.51 \pm 0.08$ \\
$Z_{\text {tot }}\left(\mathrm{nm}^{-2}\right)$ & $+1.5 \pm 0.1$ & $-2.2 \pm 0.5$ \\
\hline
\end{tabular}

The transmission electron microscopy (TEM) pictures of the two diluted ferrofluids (Figure S2) are similar and show that the shape of the magnetic nanoparticles is roughly spherical with diameters ranging between 4 and $20 \mathrm{~nm}$. Thus, the presence of citrate ligands added after IONP synthesis does not change the particle size and morphology. This is different from a drastic decrease of IONP size if trisodium citrate is added in the beginning of the synthesis. ${ }^{19} \mathrm{X}$-ray diffraction (XRD) patterns, analyzed in the Supporting Information (SI, Section A), reveal the maghemite structure of synthesized IONPs, whose crystalline phase seems not to be altered by the citrate coating in the case of FFCit samples (Figure S3).

Magnetization curves are presented in Figure S4 and were used to determine the saturation magnetization of IONPs and the average diameter of metal oxide cores of IONP, which are approximately the same for FFA and FFCit samples and equal to $M_{\mathrm{S}}=(3.1 \pm 0.1) \times 10^{5} \mathrm{~A} / \mathrm{m}$ and $d_{\mathrm{M}}=7.6-7.8 \mathrm{~nm}$, respectively. The geometric specific area of noncitrated (FFA) IONP, estimated by modeling each particle as a sphere with a diameter $d_{\mathrm{M}}$, is equal to $S \approx 152 \mathrm{~m}^{2} / \mathrm{g}$. For citrated IONP (FFCit), we used the "outer" diameter of the citrate-coated IONP, which was evaluated as $d_{\mathrm{O}}=d_{\mathrm{M}}+2 \delta_{0}=8.0-9.6 \mathrm{~nm}$ taking the thickness of the adsorbed citrate layer within the range $\delta_{0}=0.2-1 \mathrm{~nm}$ corresponding to two limiting conformations on the IONP surface. ${ }^{16,20}$ This gave the geometric specific area $S \approx 123-148 \mathrm{~m}^{2} / \mathrm{g}$ for citrated IONPs. This large specific surface area affords a high number of active sites on the nanoparticles, leading to a large dye loading capacity. Dynamic light scattering (DLS) measurements performed on the two diluted ferrofluids $\left(c_{\mathrm{w}} \sim 0.2 \mathrm{~g} / \mathrm{L}\right)$ show no particle aggregates even after more than several months. The hydrodynamic diameters are equal to $d_{\mathrm{H}}=21$ and $22 \mathrm{~nm}$ for the bare and citrate-coated IONPs, respectively.

Evidence of citrate binding to the IONP surface of the FFCit sample was provided by Fourier transform infrared (FTIR) spectra (Figure S5a), whose analysis is presented in Section A of the SI. The amount of citrate bound to the IONP was determined by thermogravimetric analysis (TGA). The TGA curve (Figure S6) shows a weight loss of $9.0 \pm 0.2 \%$ between 110 and $350{ }^{\circ} \mathrm{C}$, which was assigned to thermal degradation of citrate adsorbed onto the surface of IONP. The sample contains about 4 wt \% water, 87 wt \% iron oxide, and 9 wt \% citrate; thus, the weight ratio of bound citrate to iron oxide is $103 \pm 2 \mathrm{mg} / \mathrm{g}$ or $Q_{\text {cit }}=0.55 \pm 0.01 \mathrm{mmol} / \mathrm{g}$, which corresponds to the number density of adsorbed citrates about $Z_{\text {cit }}=2.1 \mathrm{~nm}^{-2}$. This value is higher than those reported for the adsorption of citric acid on hematite ${ }^{21}\left(Z_{\text {cit }} \sim 0.7 \mathrm{~nm}^{-2}\right.$ or 15 $\mu \mathrm{mol} / \mathrm{g}$ for $\left.S=12.7 \mathrm{~m}^{2} / \mathrm{g}\right)$ and goethite ${ }^{22}\left(Z_{\text {cit }} \sim 0.85 \mathrm{~nm}^{-2}\right.$ or $100 \mu \mathrm{mol} / \mathrm{g}$ for $\left.S=70 \mathrm{~m}^{2} / \mathrm{g}\right)$ at similar $\mathrm{pH}(\sim 7-7.7)$. The 

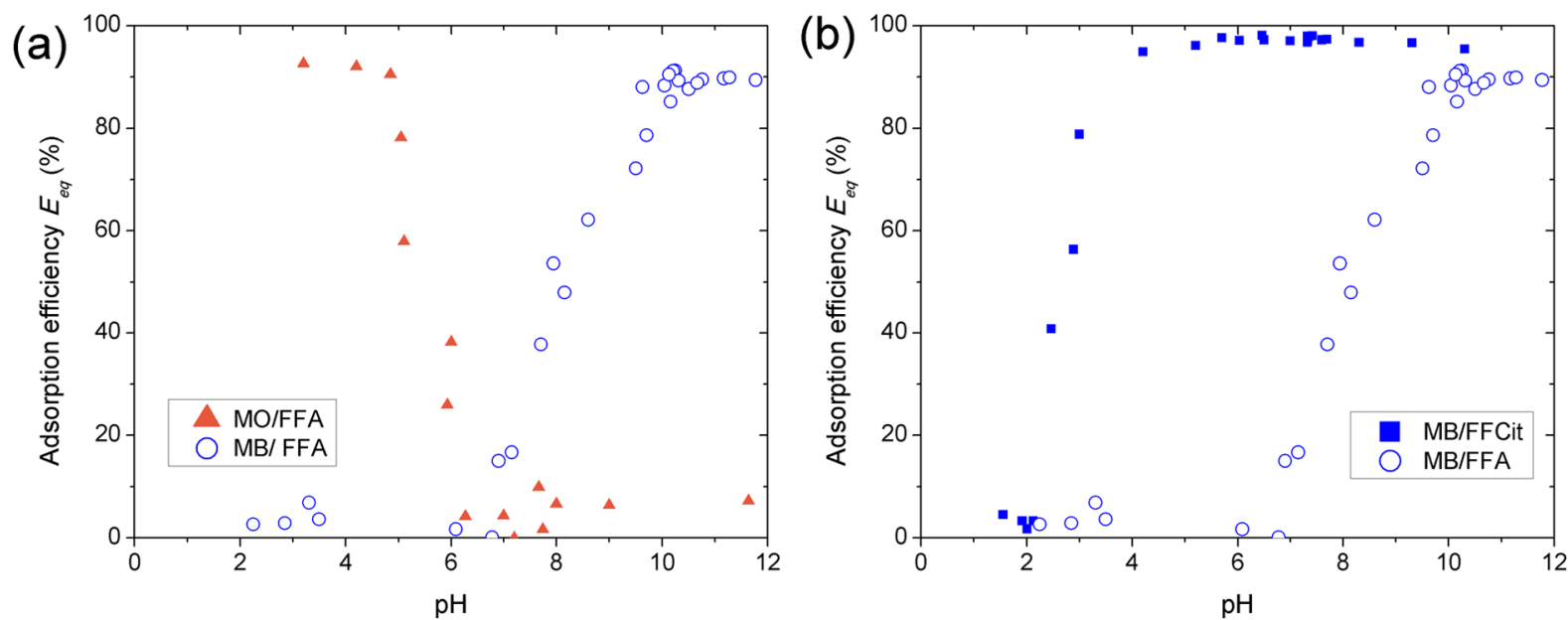

Figure 1. Effect of $\mathrm{pH}$ on adsorption efficiency: (a): MB/FFA (blue) and MO/FFA (orange); (b) MB/FFCit (squares) and MB/FFA (circles); $C_{0}$ $=0.4 \mathrm{mmol} / \mathrm{L}$ and $c_{\mathrm{w}}=2 \mathrm{~g} / \mathrm{L}$.

adsorption area $1 / 2.1 \sim 0.5 \mathrm{~nm}^{2}$ in our case lies between two limits corresponding to the linear arrangement of the carboxyl groups when the molecule is extended over the surface ${ }^{23}(\sim 0.8$ $\mathrm{nm}^{2}$ ) or vertical orientation with the only carboxylate group bonded to the surface $\left(\sim 0.3 \mathrm{~nm}^{2}\right)$. The citrate conformation on our IONP is expected to be intermediate between both these limits.

The colloidal stability of FFA ferrofluids (bare IONP) is ensured by electrostatic repulsion between charged nanoparticles. The method to determine the structural charge of the IONP depends on the nature of the ferrofluid. For bare IONPs, the surface charge is due to the acid-base properties of surface hydroxyl groups. The surface charge is positive for a $\mathrm{pH}$ value below the point of zero charge (PZC) and negative for $\mathrm{pH}$ above $\mathrm{PZC}$, leading to stable ferrofluids. The ferrofluid flocculates for a $\mathrm{pH}$ value close to $\mathrm{PZC}$ located around $\mathrm{pH} 7.5$ for maghemite. The variation of the structural charge of IONP with $\mathrm{pH}$ had been estimated from both conductimetric and potentiometric measurements (Figure S7). For the parent ferrofluid FFA whose $\mathrm{pH}$ is around 2, the nanoparticles are positively charged and the density of protonated hydroxyl groups is equal to $Q_{\text {tot }}=0.39 \mathrm{mmol} / \mathrm{g}$ (Figure S7), which corresponds to the surface density of the structural charge $Z_{\text {tot }}$ $=+1.5 \mathrm{~nm}^{-2}$.

For FFCit, the colloidal stability is ensured by electrosteric repulsion between negatively charged citrate ions adsorbed onto the IONP surface. The molecular structure of trisodium citrate (Figure S1e) shows that citrate has three carboxylate functions. Their $\mathrm{p} K_{\mathrm{a}}$ values were determined by $\mathrm{HCl}$ acid titration in the working range of ionic strength, and the obtained values, $\mathrm{p} K_{\mathrm{a} 1}=2.79, \mathrm{p} K_{\mathrm{a} 2}=4.34$, and $\mathrm{p} K_{\mathrm{a} 3}=5.83$, agree with those reported by Hidber et al. ${ }^{23}$ The ionization curve corresponding to these $\mathrm{p} K_{\mathrm{a}}$ values is shown in Figure S1f. The adsorption of citrate ions on IONP occurs via a surface complexation involving surface iron and a part of the carboxylate functional groups of citrate ions, which replace the hydroxyl groups. The remaining free carboxylate groups are responsible for the surface charge of IONP. At the $\mathrm{pH}$ value of FFCit (7.6), almost all free carboxylate groups of adsorbed citrate ions are deprotonated (as follows from Figure S1f), leading to a stable ferrofluid. By adding an alkaline solution, the ferrofluid remains stable up to $\mathrm{pH} 11$. Beyond this value, a competition occurs between hydroxyl groups and citrate ions.
In an acid medium $(\mathrm{pH}<4.5)$, protonation of adsorbed citrate induces a decrease of surface charge and the ferrofluid flocculates. The structural surface charge of the citrate-coated nanoparticles is deduced from the amount of sodium counterions determined by atomic absorption spectrometry (AAS) and confirmed by inductive coupled plasma atomic emission spectrometry (ICP-AES): $Q_{\text {tot }}=0.51 \pm 0.08 \mathrm{mmol} / \mathrm{g}$. If we compare the structural charge density $Q_{\text {tot }}$ with the amount of adsorbed citrate ions obtained by TGA $\left(Q_{\mathrm{Cit}} \approx 0.55\right.$ $\mathrm{mmol} / \mathrm{g}$ ), we obtain a ratio close to 1 , indicating that among the three carboxylate functions of an adsorbed citrate ion, two carboxylate functions are involved in the binding of the citrate ion to the nanoparticle. The remaining one free carboxylate per citrate ion ensures the negative charge of nanoparticles. This seems to not contradict the above statement on intermediate surface conformation of the citrate ions between the fully extended horizontal and fully extended vertical conformations. Similar bonding of citric acid by two carboxylic groups to the hematite surface has been reported by Kallay and Matijevic. ${ }^{24}$ These free carboxylates represent the adsorption sites of IONP for pollutants. The structural charge could also be expressed per unit surface, $Z_{\text {tot }}=-(2.2 \pm 0.5) \mathrm{nm}^{-2}$. The effective charge density $Z_{\text {eff }} \approx-0.1 \mathrm{~nm}^{-2}$ evaluated from electrophoretic mobility measurements is considerably lower (in absolute value) because of possible condensation of sodium counterions around the charged nanoparticle surface, as will be discussed in Section 2.3. Notice that citrate adsorption equilibrium imposes the presence of free citrate ions in the solvent. The amount of free citrates (revealed by $\mathrm{HCl}$ acid titration) in most of our samples corresponds to the plateau of the citrate adsorption isotherm reported by Dubois et al. ${ }^{25}$ This ensures the optimal colloidal stability of the citrate-coated IONP at neutral and basic $\mathrm{pH}$.

2.2. Dye Adsorption/Desorption Studies. The study of methylene blue (MB) and methyl orange (MO) adsorption onto the surface of IONPs dispersed in aqueous solvents was performed in a batch mode according to the protocol described in Section 4.4. The adsorption properties of IONPs are strongly affected by the solution $\mathrm{pH}$. Thus, in the beginning, we have investigated the $\mathrm{pH}$ effect on the dye adsorption efficiency $E_{\text {eq }}$ (introduced in Section 4.4) in a $\mathrm{pH}$ range from 2 to 12 . At constant IONP weight concentration $c_{\mathrm{w}}$ $=2 \mathrm{~g} / \mathrm{L}$ and total dye concentration $C_{0}=0.4 \mathrm{mmol} / \mathrm{L}$, the 
adsorption efficiency is proportional to the surface density of the adsorbed dye molecules, and the dependencies $E_{\mathrm{eq}}(\mathrm{pH})$ plotted in Figure 1 reflect the $\mathrm{pH}$ effect on the amount of adsorbed dye on the IONP surface.

In the case of FFA (Figure 1a), when IONPs are positively charged ( $\mathrm{pH}$ values lower than 7 ), the highest amount of adsorbed molecules occurs for the negatively charged MO dye, while the electrostatic repulsion between the positively charged $\mathrm{MB}$ and IONP prevents its adsorption. When the $\mathrm{pH}$ value increases above the PZC $\left(\mathrm{pH}_{\mathrm{PZC}} \sim 7.5\right.$, Figure $\left.\mathrm{S} 7\right)$, the surface charge of IONP becomes negative and the adsorption of $\mathrm{MB}$ increases, while the electrostatic repulsion induces a desorption of MO. Around the PZC, the adsorption is almost zero for both dyes. This allows concluding that electrostatic interactions between dye molecules and the bare IONP surface are dominant over other possible interactions.

In the case of FFCit (Figure $1 \mathrm{~b}$, squares), $\mathrm{MB}$ adsorption on citrate-coated nanoparticles occurs at a lower $\mathrm{pH}$ than with naked nanoparticles with $95 \%$ adsorption at $\mathrm{pH}=4.2$. Above this $\mathrm{pH}$ value, the free carboxylate functions of the adsorbed citrate are progressively deprotonated, inducing strong electrostatic interactions with the positively charged dye. For $\mathrm{pH}<4$, the carboxylate protonation becomes significant (ionization degree decreases to $43 \%$ at $\mathrm{pH}=4$, as follows from Figure S1f) and the $\mathrm{MB}$ adsorption progressively decreases with a decrease of $\mathrm{pH}$. No adsorption with protonated carboxylate occurs at $\mathrm{pH} \leq 2$.

In summary, by controlling the $\mathrm{pH}$ of the solution and the nature of the IONP surface, positively and negatively charged species can be selectively adsorbed. With naked nanoparticles, positively charged pollutants can be selectively adsorbed for $\mathrm{pH}>7$ and negatively charged pollutants can be selectively adsorbed in an acid medium. On the other hand, the citrated nanoparticles adsorb only negative species for $\mathrm{pH}>4$.

The kinetics of dye adsorption by IONP was also investigated. Figure S9 illustrates the effect of contact time on the adsorption of dyes. The adsorption is fast, and in all cases, the equilibrium is reached in less than $30 \mathrm{~min}$. Since magnetic nanoparticles are not porous, the adsorption sites are easily accessible to dye molecules. The quantities adsorbed at equilibrium for the chosen initial dye concentrations agree with the adsorption isotherms described below. In view of these results, for all experiments carried out at equilibrium, the reaction time chosen will be at least $1 \mathrm{~h}$, which is enough to reach equilibrium.

The adsorption isotherms of MB on the surface of IONPs with or without citrate coating and MO on the surface of bare IONPs are shown in Figure 2. The weight concentration of IONP, $c_{w}$, is $2 \mathrm{~g} / \mathrm{L}$, and the measurements were carried out at a $\mathrm{pH}$ value (listed in Table 2) for which adsorption is the most efficient. In the three cases, the amount of adsorbed dye increases with increasing concentration of dye until reaching a plateau, which corresponds to the maximum adsorption capacity of IONPs. This maximum is explained by a definite quantity of active sites on IONPs. The experimental data are satisfactorily fitted by the single Langmuir-type isotherm. ${ }^{26}$ Its linearized form is $C_{\mathrm{eq}} / Q_{\mathrm{eq}}=1 /\left(Q_{\max } K_{\mathrm{L}}\right)+C_{\mathrm{eq}} / Q_{\max }$ where $Q_{\mathrm{eq}}$ is the amount of adsorbed dye at equilibrium, $Q_{\max }$ is the maximum adsorption capacity corresponding to the plateau of the adsorption isotherm, $C_{\mathrm{eq}}$ is the concentration of the dye in the solution at equilibrium, and $K_{\mathrm{L}}$ is the Langmuir constant. Linearized Langmuir plots are presented in Figure S10. Table 2 reports the values of fitting parameters $Q_{\max }$ and $K_{\mathrm{L}}$. Langmuir
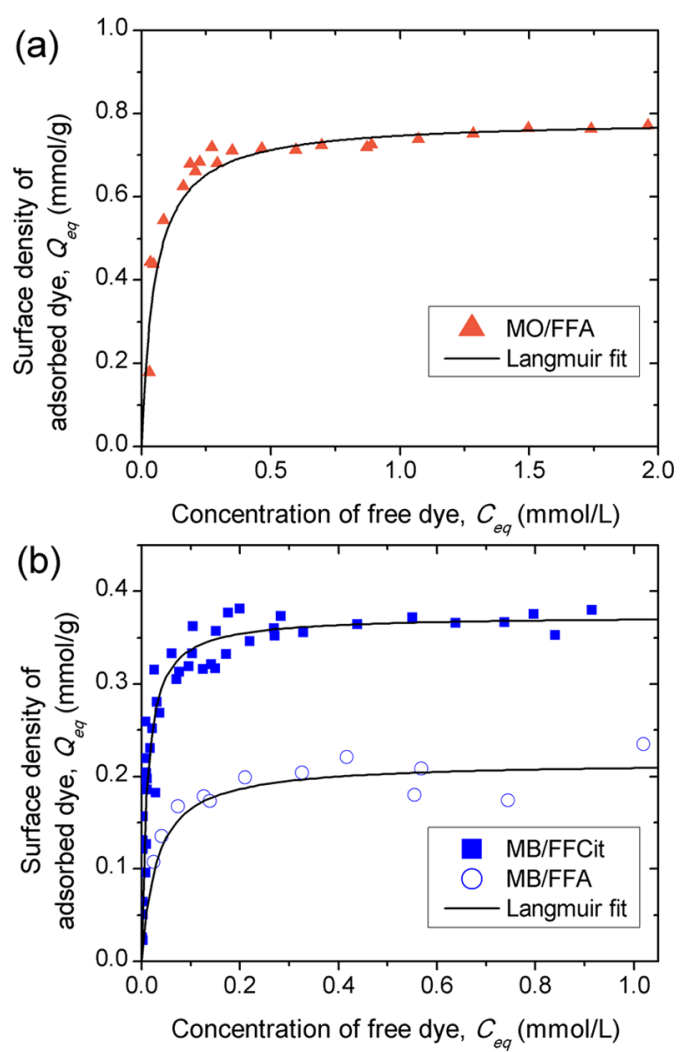

Figure 2. Adsorption isotherms of dyes on IONPs for MO (a) and $\mathrm{MB}$ (b). Experimental data are represented by symbols, and the fit by the Langmuir adsorption law is represented by solid black lines. The IONP weight concentration is $c_{\mathrm{w}}=2 \mathrm{~g} / \mathrm{L}$. The $\mathrm{pH}$ values are reported in Table 2.

constants $K_{\mathrm{L}}$ of $\mathrm{MO} / \mathrm{FFA}$ and $\mathrm{MB} / \mathrm{FFA}$ systems are quite close, indicating a nearly similar energy of interaction between the dye molecule and surface adsorption sites. On the other hand, when $\mathrm{MB}$ adsorptions onto bare (FFA) and citrated (FFCit) IONPs are compared, the interaction energy is clearly higher for citrated IONPs likely because of the stronger electronegativity of free carboxylate groups of bound citrate than hydroxyl groups present on the surface of bare IONPs.

For further analysis, we also present in Table 2 the maximum adsorption capacity in terms of the maximal number density $Z_{\max }=Q_{\max } N_{\mathrm{A}} / S$ of adsorbed dye molecules. Possible mechanisms of adsorption will be discussed in Section 2.3 for the MB/FFCit sample, but we can already notice the following points. In the case of FFA, the $Q_{\max }$ value obtained for $\mathrm{MB}$ adsorption on naked IONP $\left(Q_{\max }=0.22 \pm 0.02 \mathrm{mmol} / \mathrm{g}\right.$ or $\left.Z_{\max }=0.85 \pm 0.08 \mathrm{~nm}^{-2}\right)$ is close to the amount of available negatives sites at $\mathrm{pH} 9.8\left(Q_{\text {tot }} \sim 0.22 \mathrm{mmol} / \mathrm{g}\right.$ or $\left|Z_{\text {tot }}\right| \sim 0.85$ $\left.\mathrm{nm}^{-2}\right)$ as inferred from potentiometric and conductimetric measurements (Figure S7). This is in agreement with a $1: 1$ ionic exchange between $\mathrm{MB}$ and the nitrate counterions of IONP and corresponds to the dense packing of $\mathrm{MB}$ molecules oriented horizontally on the IONP surface, i.e., with the largest B face (of an area $1.3 \mathrm{~nm}^{2}$, cf. ref 27, Figure S1b) adsorbed onto the surface of bare IONPs. For MO, it is quite different: $Q_{\max }=0.78 \pm 0.05 \mathrm{mmol} / \mathrm{g}$ and $Z_{\max }=3.1 \pm 0.2 \mathrm{~nm}^{-2}$ are much greater than the number of available sites $Q_{\text {tot }}=0.33 \pm$ $0.05 \mathrm{mmol} / \mathrm{g}$ and $Z_{\text {tot }}=1.3 \pm 0.2 \mathrm{~nm}^{-2}$, as inferred from Figure $\mathrm{S} 7$ at $\mathrm{pH}=3.9 \pm 0.07$. Some hypotheses are proposed to explain this. First, we observed a precipitation of $\mathrm{MO}$ in 
Table 2. Langmuir Parameters and Surface Density of Adsorbed Dyes $\left(Q_{\max }\right.$ and $\left.Z_{\max }\right)$ vs Surface Density of Available Adsorption Sites $\left(Q_{t o t}\right.$ and $\left.\left|Z_{t o t}\right|\right)$

$\begin{array}{lcccccccc}\text { FF } & \text { dye } & \mathrm{pH} & Q_{\max }(\mathrm{mmol} / \mathrm{g}) & Q_{\max }(\mathrm{mg} / \mathrm{g}) & Z_{\max }\left(\mathrm{nm}^{-2}\right) & K_{\mathrm{L}}(\mathrm{L} / \mathrm{mmol}) & Q_{\text {tot }}(\mathrm{mmol} / \mathrm{g}) & \left.\left|Z_{\text {tot }}\right|(\mathrm{nm})^{-2}\right) \\ \text { FFA } & \text { MO } & 3.9 \pm 0.7 & 0.78 \pm 0.05 & 257 \pm 16 & 3.1 \pm 0.2 & 20 & 0.33 \pm 0.05^{a} & 1.3 \pm 0.2 \\ \text { FFA } & \text { MB } & 9.8 \pm 0.1 & 0.22 \pm 0.02 & 81 \pm 8 & 0.85 \pm 0.08 & 32 & \sim 0.22^{a} & \sim 0.85 \\ \text { FFCit } & \text { MB } & 7.5 \pm 0.2 & 0.37 \pm 0.01 & 139 \pm 4 & 1.6 \pm 0.2 & 93 & 0.51 \pm 0.08 & 2.2 \pm 0.5\end{array}$

${ }^{a_{T}}$ The values of $Q_{\text {tot }}$ for MO/FFA and MB/FFA samples are taken from Figure $\mathrm{S} 7$ at the $\mathrm{pH}$ values reported on the third column of this table. These values of $Q_{\text {tot }}$ are therefore different from the value $Q_{\text {tot }}=0.39 \mathrm{mmol} / \mathrm{g}$ reported in Table 1 for $\mathrm{pH} \sim 2$.

Milli-Q water without IONPs probably due to dye aggregation (likely promoted by aromatic short-ranged interactions). The $\mathrm{pH} /$ concentration ranges where precipitation was observed sometimes overlapped with those of the adsorption isotherm. The formation of these aggregates would affect the dosage of MO in the supernatant by underestimating its value, which could explain the high value of $Q_{\max }$. Another possibility is to suppose that, geometrically, the obtained surface density $Z_{\max }$ corresponds to a dense packing of $\mathrm{MO}$ with the aromatic rings oriented perpendicularly to the IONP surface. This corresponds to the molecular adsorption area $\sim 1 / Z_{\max } \sim 0.3 \mathrm{~nm}^{2}$ situated between geometric areas $\sim 0.13-0.28 \mathrm{~nm}^{2}$ and $\sim 0.43-0.48 \mathrm{~nm}^{2}$ of two MO faces perpendicular to aromatic rings. $^{28,29}$ We can speculate that the maximal adsorption capacity is limited by steric hindrance between MO molecules rather than by the number of available adsorption sites. This is likely thanks to attractive $\pi$-stacking interactions between MO molecules promoting their lateral aggregation on the IONP surface (as often observed during dye adsorption ${ }^{30-32}$ ) and increasing adsorption capacity. Nevertheless, a more detailed study is required to further elucidate the discrepancy between $Q_{\max }$ and $Q_{\text {tot }}$ values for MO adsorption on bare IONPs.

For FFCit, the $Q_{\max }$ value obtained for $\mathrm{MB}\left(Q_{\max }=0.37 \pm\right.$ $\left.0.01 \mathrm{mmol} / \mathrm{g}, Z_{\max }=1.6 \pm 0.2 \mathrm{~nm}^{-2}\right)$ is lower than the estimated adsorption sites of citrate-coated IONPs $\left(Q_{\text {tot }}=0.51\right.$ $\pm 0.08 \mathrm{mmol} / \mathrm{g},\left|Z_{\text {tot }}\right|=2.2 \pm 0.5 \mathrm{~nm}^{-2}$ ), and this issue will be inspected in detail in Section 2.3 in close relation with the study of IONP agglomeration induced by MB adsorption.

From a practical perspective, reusability of magnetic nanoparticles is one of the key factors in evaluating the performance of IONPs as dye adsorbents. To investigate this reusability, an adsorption/desorption cycle was realized 9 times for the system MB/FFCit using an initial $\mathrm{MB}$ concentration equal to $C_{0}=4 \mathrm{mmol} / \mathrm{L}$ at $c_{\mathrm{w}} \sim 20 \mathrm{~g} / \mathrm{L}$. Adsorption was realized at $\mathrm{pH}=8.1$ and desorption at $\mathrm{pH}=$ 1.8 , nitric acid being used as the desorption agent. Its function is to protonate the carboxylate of the adsorbed citrate to substantially reduce the electrostatic interaction between $\mathrm{MB}$ and IONP. As it can be seen in Figure 3, the amount of desorbed MB varies between 61 and $79 \%$, where we expected almost $100 \%$ with regard to Figure $1 \mathrm{~b}$. At this moment, we do not have a clear explanation for incomplete MB desorption. Reducing the $\mathrm{pH}$ further can promote dye desorption but induce dissolution of the nanoparticles. However, the compromise chosen is acceptable because the adsorption remains greater than $90 \%$ even after nine adsorption/ desorption cycles. This result shows that the citrate-coated IONPs could be used in a water treatment process without significant losses of their adsorption efficiency. It is worth noticing that, apart from $\mathrm{pH}$ reversal, the IONP regeneration could likely be realized by transferring nanoparticles from an aqueous solvent to an organic one. Such a technique allowed more than $80 \%$ regeneration efficiency after multiple $\mathrm{MB}$

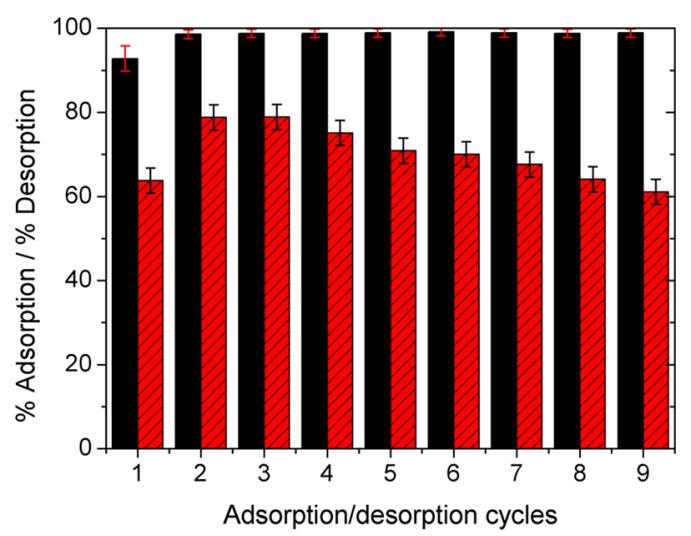

Figure 3. Cycles of successive adsorption (black) and desorption (red) of MB on citrate-coated IONPs (MB/FFCit samples) at the initial $\mathrm{MB}$ concentration $C_{0}=4 \mathrm{mmol} / \mathrm{L}$, IONP weight concentration $c_{\mathrm{w}} \sim 20 \mathrm{~g} / \mathrm{L}$, reaction time $30 \mathrm{~min}$, adsorption $\mathrm{pH}=8.1 \pm 0.4$, and desorption $\mathrm{pH}=1.85 \pm 0.04$.

adsorption/desorption cycles using acetone and acetonitrile solvents as desorbing agents and graphene/wood composites as sorbent materials. ${ }^{3}$

Notice finally that the values of the maximum adsorption capacity $Q_{\max }$ reported in Table $2(257 \mathrm{mg} / \mathrm{g}$ for $\mathrm{MO}$ and $81-$ $139 \mathrm{mg} / \mathrm{g}$ for MB) are in general comparable to those reported in the literature on adsorption of $\mathrm{MO}$ and $\mathrm{MB}$ onto iron oxidebased nanocomposites: for instance, $152 \mathrm{mg} / \mathrm{g}$ for $\mathrm{MO}$ adsorption onto porous carbon/IONP composites, 48-62 $\mathrm{mg} / \mathrm{g}$ for $\mathrm{MB}$ adsorption onto carbon nanotube/IONP composites, and $93 \mathrm{mg} / \mathrm{g}$ for $\mathrm{MB}$ adsorption onto humic acid-coated IONP (see reviews by Tan et al. ${ }^{34}$ and Gupta et $\mathrm{al}^{35}$ and the references therein), $73 \mathrm{mg} / \mathrm{g}$ for MB adsorption onto polyoxometalate/IONP hybrids ${ }^{36,37}$ and $14 \mathrm{mg} / \mathrm{g}$ for MB adsorption onto cellulose-capped IONPs, ${ }^{38}$ although chitosanbased magnetic composites offer a higher adsorption capacity: $750 \mathrm{mg} / \mathrm{g}$ for $\mathrm{MO}^{39}$ and $2800 \mathrm{mg} / \mathrm{g}$ for MB (see the review by Kyzas et al. ${ }^{40}$ and the references therein).

2.3. Focus on $\mathrm{MB}$ Adsorption and AdsorptionInduced Agglomeration of Citrate-Coated IONPs. The very important issue that cannot be ignored while considering adsorption is nanoparticle agglomeration induced by adsorption (primary agglomeration). On the one hand, MB adsorption on citrate-coated IONPs is expected to promote attractive colloidal interactions between nanoparticles inducing agglomeration. On the other hand, this agglomeration could impact the spatial distribution of adsorbed MB molecules and somehow influence the adsorption equilibrium. Finally, this adsorption-induced agglomeration facilitates nanoparticle aggregation (secondary agglomeration) and magnetic separation in the presence of a magnetic field, as will be discussed in detail in the companion paper (Part II). We start by empirical description of the primary agglomeration, and at the end of this 
Table 3. Physicochemical Characterization of the FFCit Dilute Ferrofluid Samples

\begin{tabular}{|c|c|c|c|c|c|c|c|c|c|c|c|c|}
\hline sample & $\begin{array}{c}c_{\mathrm{w}} \\
(\mathrm{g} / \mathrm{L})\end{array}$ & $\begin{array}{c}d_{\mathrm{H}} \\
(\mathrm{nm})\end{array}$ & PDI & $\mathrm{pH}$ & $\left(10^{8} \times \mathrm{m}^{2} \mathrm{~s}^{-1} \mathrm{~V}^{-1}\right)$ & $\begin{array}{c}\sigma \\
(\mathrm{mS} / \mathrm{cm})\end{array}$ & $C_{\mathrm{Na}}{ }^{a}(\mathrm{mM})$ & $\begin{array}{l}Q_{\text {tot }}=Q_{\mathrm{Na}}{ }^{a} \\
(\mathrm{mmol} / \mathrm{g})\end{array}$ & $I(\mathrm{mM})$ & $\kappa^{-1}(\mathrm{~nm})$ & $\begin{array}{c}\left|Z_{\text {tot }}\right| \\
\left(\mathrm{nm}^{-2}\right)\end{array}$ & $\begin{array}{c}\left|Z_{e f f}\right| \\
\left(\mathrm{nm}^{-2}\right)\end{array}$ \\
\hline SD & 0.18 & 22 & 0.23 & 6.50 & -3.3 & 0.074 & $0.60 \pm 0.10$ & $0.51 \pm 0.05$ & $1.2 \pm 0.2$ & $8.8 \pm 0.8$ & $2.2 \pm 0.4$ & 0.12 \\
\hline SC & 8.0 & $\mathrm{~N} / \mathrm{A}$ & N/A & 7.60 & -3.2 & 2.36 & $24.9 \pm 0.1$ & $0.57 \pm 0.03$ & $49.8 \pm 0.2$ & $1.5 \pm 0.1$ & $2.5 \pm 0.3$ & 0.23 \\
\hline
\end{tabular}

section, we will try to understand its reasons by a more careful inspection of the adsorption mechanisms.

These studies have been carried out with the diluted ferrofluids of weight concentrations $c_{\mathrm{w}}=0.18 \mathrm{~g} / \mathrm{L}$ (sample SD) and $8 \mathrm{~g} / \mathrm{L}$ (sample SC). The lowest concentration allowed a reliable hydrodynamic size and electrophoretic mobility measurements, while the highest concentration allowed reliable determination of sodium and citrate concentrations in the solvent and was used in magnetic separation studies (cf. companion paper, Part II). All relevant characterizations of the samples SD and SC are summarized in Table 3. Adsorption isotherms were remeasured for the samples SD and SC within the range of free $\mathrm{MB}$ concentration $C_{\mathrm{eq}}=0-0.20 \mathrm{mmol} / \mathrm{L}$, relevant for these experiments. We observe that within experimental errors, the adsorption isotherm is similar for three considered concentrations $c_{\mathrm{w}}=0.18,2$, and $8 \mathrm{~g} / \mathrm{L}$ (Figure S11). We believe therefore that the concentration difference between different data of this section should not affect the main conclusions.

To proceed, we introduce the surface coverage $\theta=Q_{\mathrm{eq}} / Q_{\max }$ as a dimensionless counterpart of the surface density $Q_{\mathrm{eq}}$ of adsorbed MB molecules. The primary agglomeration of IONP as a result of the $\mathrm{MB}$ adsorption was tested through the hydrodynamic size distribution measurements shown in Figure 4 a for the SD-MB samples for different surface coverages $\theta$ and at elapsed time equal to $1 \mathrm{~h}$ after the end of the mixing of $\mathrm{MB}$ and IONP solutions. The size distribution appears to progressively shift to the higher hydrodynamic sizes with increasing surface coverage, and initially monomodal distribution in the absence of $\mathrm{MB}$ becomes multimodal with increasing $\theta$. The $Z$-average diameter and the polydispersity index (PDI) are shown in Figure $4 \mathrm{~b}$ as a function of the surface coverage or surface density of adsorbed MB. The $Z$-average size exhibits an exponential increase with $\theta$, clearly observed in the semi-log scale: $d_{\mathrm{H}}=d_{\mathrm{H} 0} \exp \left(\alpha_{0} \theta\right)$, with $d_{\mathrm{H} 0}=17 \pm 5 \mathrm{~nm}$ and $\alpha_{0}=6.0 \pm$ 0.3 - two fitting parameters, the first of which $\left(d_{\mathrm{HO}}\right)$ is the $Z$ average size in the absence of MB. Notice that the experimental value of the hydrodynamic size in the absence of MB (22 nm, cf. Table 3) enters the confidence interval of the above value of $d_{\mathrm{H} 0}$ obtained by the fit. Such a behavior along with a pronounced increase of the PDI with $\theta$ clearly indicates the agglomeration of IONPs with increasing amounts of adsorbed MB. Such an agglomeration could result either in dense dropletlike aggregates, as often reported for IONPs with screened electrostatic repulsion, ${ }^{41,42}$ or in fractal clusters. Nevertheless, for any of these morphologies, the hydrodynamic radius $d_{\mathrm{H}} / 2$ of the agglomerates revealed by DLS is relatively close to their gyration radius, ${ }^{43}$ such that $d_{\mathrm{H}}$ can still be associated with the geometric size. Experiments also show that at $\theta<54 \%$, the hydrodynamic size of agglomerates increases drastically during mixing of $\mathrm{MB}$ and IONP solutions and does not evolve substantially with time for $3 \mathrm{~h}$ after the end of $\mathrm{MB}$ / IONP stirring but only evolves on the scale of a few days (results not shown for brevity). This can be associated with a (a)
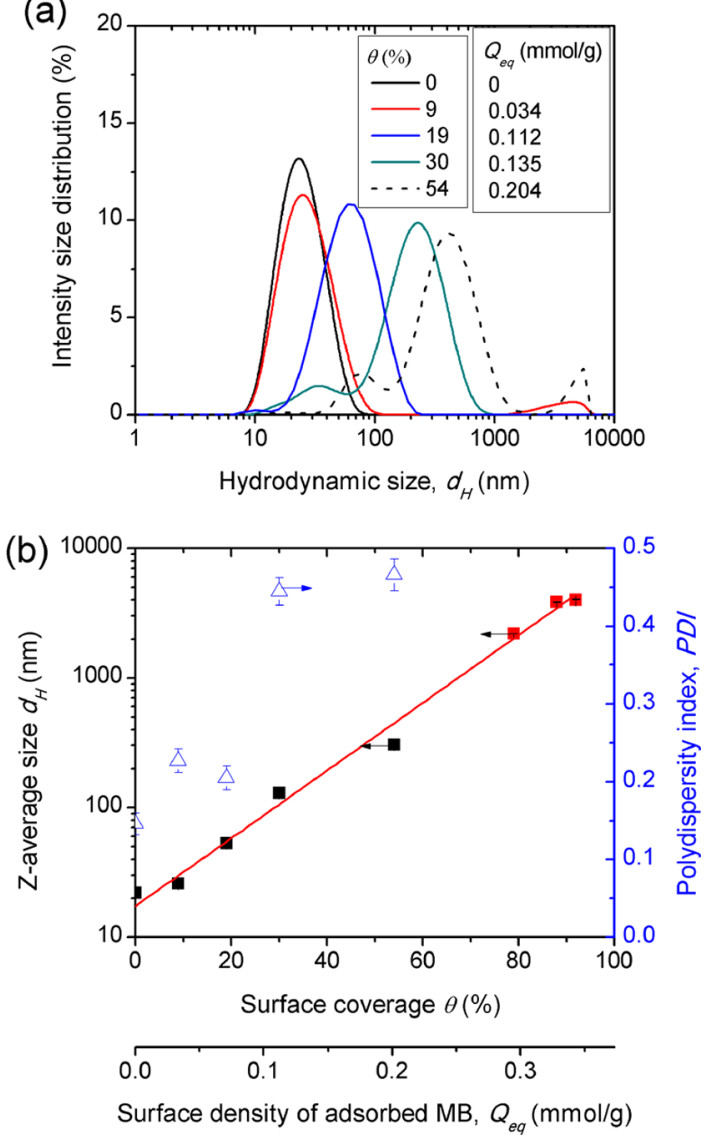

Figure 4. Hydrodynamic size distribution of primary agglomerates measured in SD-MB samples by DLS (a) and dependencies of the $Z$ average size (on the left ordinate axis) and PDI (on the right ordinate axis) on the surface coverage/surface density of adsorbed MB (b). The red line in (b) stands for the exponential fit of the $d_{\mathrm{H}}(\theta)$ dependency. The points of the $d_{\mathrm{H}}(\theta)$ dependency corresponding to highly agglomerated samples are marked in red; the DLS measurements are subjected to large errors for these points.

faster shear-induced flocculation during stirring than Brownian flocculation after stirring.

At first glance, the most intuitive reason for the IONP primary agglomeration induced by $\mathrm{MB}$ adsorption is related to a decrease of effective electric charge on the negatively charged IONP surface when positively charged MB species adsorb onto this surface. To check this hypothesis, we measured the electrophoretic mobility of IONP agglomerates in the presence of adsorbed $\mathrm{MB}$ and converted it into the effective charge density on the surface of individual nanoparticles constituting agglomerates using the model of Miller et al. ${ }^{44}$ (SI, Section C). The electrophoretic mobility $\mu_{\mathrm{E}}$ (the left ordinate axis) and the effective charge density $Z_{\text {eff }}$ (the right ordinate axis) are plotted as functions of either the surface concentration of adsorbed $M B Q_{\text {eq }}$ or the surface coverage $\theta$ in Figure 5 a for the SD-MB samples. We remark that, in contrast to what was expected, $Z_{\text {eff }}$ 

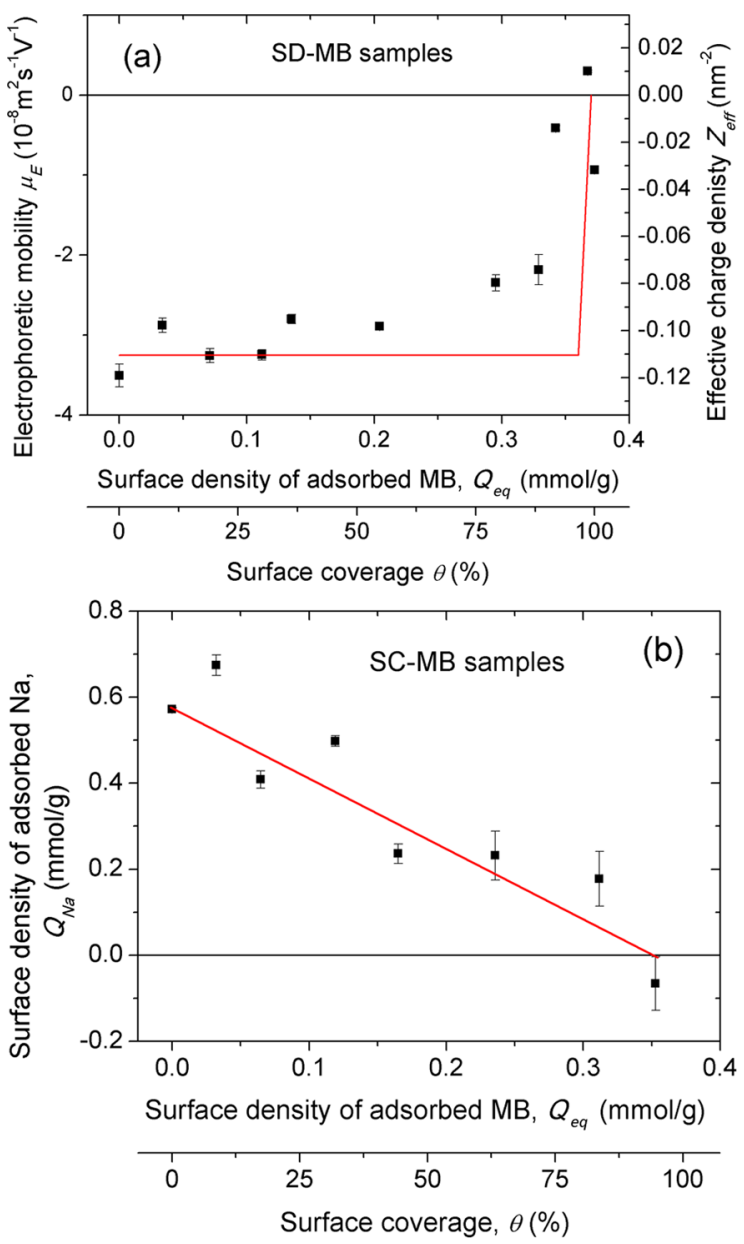

Figure 5. Dependencies of the electrophoretic mobility and the effective charge density (a) as well as of the surface density of the sodium counterions adsorbed (condensed) on the IONP surface (b) on the surface coverage/surface density of the adsorbed MB. Experiments are realized on SD-MB samples (a) and SC-MB samples (b). The solid red line in (a) corresponds to the prediction of the counterion condensation theory. The solid red line in (b) stands for the linear fit of the surface density of adsorbed (condensed) sodium counterions vs surface density of adsorbed MB molecules.

experiences only a slight variation over the wide range of surface coverage $0 \leq \theta<60 \%$, and its absolute value decreases to a near-zero value only at $\theta$ close to $100 \%$. Furthermore, the value of the effective charge $\left|Z_{\text {effl }}\right| \approx 0.1 \mathrm{~nm}^{-2}$ at the plateau of the $Z_{\text {eff }}(\theta)$ curve is much smaller than the value of the total (structural) charge $\left|Z_{\text {tot }}\right|=2.2 \pm 0.4 \mathrm{~nm}^{-2}$ for the SD sample or $\left|Z_{\text {tot }}\right|=2.5 \pm 0.3 \mathrm{~nm}^{-2}$ for the SC sample, as revealed by sodium dosage (Table 3 ).

Thus, the results presented in Figures 4 and 5 a raise the following three questions.

(1) How to explain the relative smallness of the effective charge $\mid Z_{\text {eff }} l$ as compared to the structural charge $\left|Z_{\text {tot }}\right|$ ?

(2) How to explain the insignificant variation of $\left|Z_{\text {eff }}\right|$ with adsorption of $\mathrm{MB}$ up to surface coverage $\theta \sim 60 \%$ ?

(3) What is the possible reason for the IONP primary agglomeration within the range $0<\theta<60 \%$, at which the surface charge (Figure 5a) and the Debye length (Figure S8) show only a slight variation with $\theta$, such that the electrostatic repulsion between IONP is expected to be almost unaltered, at least in the limit of the
Derjaguin-Landau-Verwey-Overbeek (DLVO) theor$y ?^{45}$

The first two questions can be responded considering the condensation of sodium counterions onto the citrate-coated IONP surface. In general, counterion condensation is defined as the formation of a dense counterion phase near a charged surface when the counterions do not specifically adsorb to any surface site but possess a translational freedom along this surface. ${ }^{46,47}$ In the absence of $\mathrm{MB}$, the nanoparticle effective charge density is equal to the difference of the structural charge density and the charge density of the condensed sodium counterions: $\left|Z_{\text {eff }}\right|=\left|Z_{\text {tot }}\right|-Z_{\mathrm{Na}}$. According to the counterion condensation theory, ${ }^{46}$ the counterions start condensing onto the charged surface if the structural surface charge $\left|Z_{\text {tot }}\right|$ overcomes a critical value $Z_{c}$. If $\left|Z_{\text {tot }}\right| \geq Z_{c}$, the effective particle charge remains nearly constant and equal to $\left|Z_{\text {eff }}\right| \approx Z_{c}$. If $\left|Z_{\text {tot }}\right|$ $<Z_{\mathcal{c}}$ the counterions no longer form a condensed phase, and the effective particle charge equals the total structural charge $\left|Z_{\text {eff }}\right| \approx Z_{\text {tot. }}$. The absolute value of the critical charge density for the present case of monovalent counterions condensed onto a spherical nanoparticle is evaluated through ${ }^{48} Z_{\mathrm{c}} \approx-\left(1+\kappa d_{\mathrm{O}} /\right.$ $2) \ln \left(\kappa \lambda_{\mathrm{B}}\right) /\left(\pi \lambda_{\mathrm{B}} d_{\mathrm{O}}\right)$, where $\lambda_{\mathrm{B}} \approx 0.71 \mathrm{~nm}$ is the Bjerrum length, $d_{\mathrm{O}}=8.0-9.6 \mathrm{~nm}$ is the IONP diameter comprising the citrate shell (Section 2.1), and the values of the Debye length $\kappa^{-1}$ are provided in Table 3 and Figure $S 8$. We evaluate $Z_{\mathrm{c}} \approx 0.2 \mathrm{~nm}^{-2}$ for both SC and SD samples. This value is an order of magnitude smaller than the experimental value of the structural charge $\left|Z_{\text {tot }}\right| \approx 2.2-2.5 \mathrm{~nm}^{-2}$ and suggests counterion condensation. The theoretical value $\left(\mid Z_{\text {eff }} \approx Z_{\mathrm{c}} \approx 0.2 \mathrm{~nm}^{-2}\right)$ of the effective charge is on the same order of magnitude as the experimental value $\mid Z_{\text {eff }} \approx 0.1 \mathrm{~nm}^{-2}$, both being much lower than the structural charge.

When MB is added to FFCit samples, it is expected to bind to the free carboxylate groups of the adsorbed citrate ions. This should reduce the number of free carboxylates available on the surface as well as the surface charge density $Z_{\mathrm{COO}}<0$ associated with free deprotonated carboxylates. The sodium condensation is likely governed by the charge density $Z_{\mathrm{COO}}$ rather than by the total structural charge $Z_{\text {tot }}$ before $\mathrm{MB}$ adsorption (notice that $Z_{\text {tot }}$ is equal to $Z_{\mathrm{COO}}$ in the absence of $\mathrm{MB}$ ). With increasing amounts $Q_{\mathrm{eq}}$ ( or $\theta$ ) of adsorbed $\mathrm{MB}$, $I Z_{\text {CoO }} \mid$ progressively decreases up to some critical value $Z_{\mathrm{c}}$ at some critical surface density $Q_{c}$ of adsorbed MB. Following Manning's theory, the sodium counterions are condensed and the effective charge density remains constant $\mid Z_{\text {eff }} \approx Z_{\mathrm{c}} \approx 0.1$ $\mathrm{nm}^{-2}$ at $Z_{\mathrm{c}} \leq\left|Z_{\mathrm{COO}}\right| \leq\left|Z_{\text {tot }}\right|$ and within the MB surface density range $0 \leq Q_{\mathrm{eq}} \leq Q_{c}$. Above the $Q_{c}$ value, the sodium counterions are expected to no longer be condensed onto the IONP surface but are present in excess in the diffuse electric double-layer. At $Q_{\mathrm{eq}}>Q_{\mathrm{o}}$, the absolute value of the effective charge density $\mid Z_{\text {eff }}$ should progressively decrease with increase of $Q_{\text {eq. }}$. This reasoning qualitatively explains the shape of the experimental curve $Z_{\text {eff }}\left(Q_{\text {eq }}\right)$ shown in Figure 5a.

For quantitative evaluation of this curve, we need to know the relationship between $\left|Z_{\mathrm{COO}}\right|$ and $Q_{\mathrm{eq}}$. At first glance, the amount of free deprotonated carboxylates is equal to the difference of the total structural charge and the quantity of adsorbed MB: $\left|Z_{\mathrm{COO}}\right|=\left|Z_{\text {tot }}\right|-Q_{\mathrm{eq}} N_{\mathrm{A}} / S$, with $N_{\mathrm{A}} \approx 6.0 \times 10^{23}$ $\mathrm{mol}^{-1}$ being the Avogadro number and $S=123-148 \mathrm{~m}^{2} / \mathrm{g}$ being the geometric specific area of citrated IONPs (Table 1 ). However, at maximum $\mathrm{MB}$ adsorption, $Q_{\mathrm{eq}}=Q_{\max }=0.37$ $\mathrm{mmol} / \mathrm{g}$, we get $\left|Z_{\mathrm{COO}}\right|=0.6-0.9 \mathrm{~nm}^{-2}$, which is substantially 

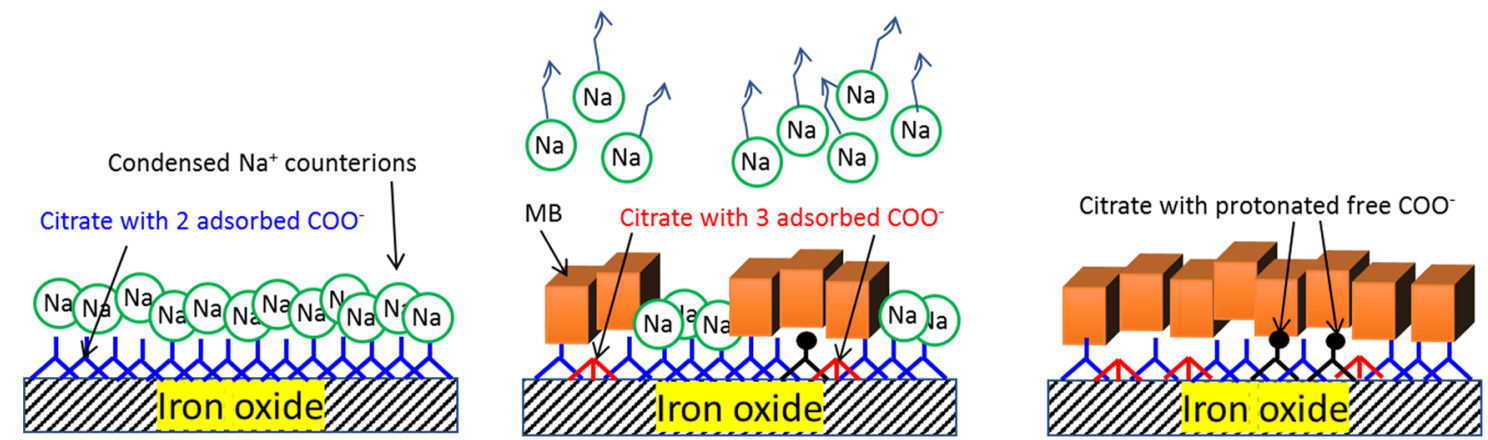

Figure 6. Schematic representation of the MB adsorption process on citrate-coated IONPs. On the left, the citrate-coated surface with a condensed layer of sodium counterions without adsorbed MB molecules is shown. In the middle, the beginning of MB adsorption is shown with formation of $\mathrm{H}$-aggregates, unbalanced 1:1.5 counterion exchange with sodium, and possible protonation or adsorption of a few free carboxylate groups of adsorbed citrate ions allowing the maintaining of a weak effective charge density $\left|Z_{\text {eff }}\right| \sim 0.1 \mathrm{~nm}^{-2}$. On the right is the final stage of the MB adsorption forming a monolayer of the densely packed molecules oriented "perpendicularly" to the surface and attached by their C-face (Figure $S 1 b, c)$ to the surface.

higher than the experimental critical surface charge density $Z_{\mathrm{c}}$ $\approx 0.1 \mathrm{~nm}^{-2}$. This implies that the sodium counterions should be in the condensed state, and the effective charge density should be constant in the whole range of the MB surface coverage, which is inconsistent with the experimental curve in Figure 5a. The value of $Z_{\mathrm{COO}}$ needs to be checked, and it can be evaluated by dosage of the condensed sodium counterions by ICP-AES, as described in Section 4.2.

The surface density $Q_{\mathrm{Na}}$ of sodium counterions condensed on the IONP surface is plotted as a function of the surface density of adsorbed $\mathrm{MB}, \mathrm{Q}_{\mathrm{eq}}$ (or the surface coverage $\theta$ ) in Figure $5 \mathrm{~b}$, for the SC-MB samples. Despite some dispersion in data (related to analytical errors), they seem to collapse onto a straight line, $Q_{\mathrm{Na}} \approx Q_{\text {tot }}-1.5 Q_{\text {eq }}$, where $Q_{\text {tot }}=0.57 \pm 0.03$ $\mathrm{mmol} / \mathrm{g}$ is the surface density of the structural charge of the SC samples (Table 3), determined by ICP-AES. A negative slope of the $Q_{\mathrm{Na}}$ vs $Q_{\mathrm{eq}}$ dependency is roughly equal to -1.5 , meaning that about 1.5 sodium ions are displaced per each adsorbing $\mathrm{MB}$ molecule. If we assume that the surface charge density $\left|Z_{\mathrm{COO}}\right|$ of deprotonated free carboxylates approximately corresponds to the amount of condensed sodium counterions, we can evaluate the critical surface density $Q_{c}$ of adsorbed $M B$ above which the effective particle charge significantly decreases to zero. This is done by equating $\left|Z_{\mathrm{COO}}\right|=Q_{\mathrm{Na}_{\mathrm{A}}} N_{\mathrm{A}} / S$ to the critical surface charge density $Z_{\mathrm{c}} \sim 0.1 \mathrm{~nm}^{-2}$. This gives us the concentration of the condensed sodium $Q_{\mathrm{Na}} \approx 0.03 \mathrm{mmol} / \mathrm{g}$ and corresponds to the concentration of adsorbed $\mathrm{MB} Q_{\mathrm{c}}=$ $\left(Q_{\text {tot }}-Q_{\mathrm{Na}}\right) / 1.5 \approx 0.36 \mathrm{mmol} / \mathrm{g}$ and to the surface coverage $\theta$ $\approx 0.97$. The theoretical dependency of $Z_{\text {eff }}\left(Q_{\text {eq }}\right)$ takes the final form as follows

$$
\left|Z_{\text {eff }}\right|=\left\{\begin{array}{l}
Z_{\mathrm{c}} \text {, at } Q_{\text {eq }} \leq Q_{\mathrm{c}} \\
\left|Z_{\mathrm{COO}}\right|=\left(Q_{\text {tot }}-1.5 Q_{\mathrm{eq}}\right) N_{\mathrm{A}} / S, \text { at } Q_{\mathrm{eq}}>Q_{\mathrm{c}}
\end{array}\right.
$$

The theoretical dependence $Z_{\text {eff }}\left(Q_{\text {eq }}\right)$ is plotted as a solid red line in Figure 5a. We see that the theory overestimates the $Q_{c}$ value likely because the concentration of condensed sodium does not reflect correctly the amount of deprotonated free carboxylates in the vicinity of $Q_{c}$.

Figure $5 \mathrm{~b}$ deserves further analysis. It reveals the counterion exchange between sodium and $\mathrm{MB}$ and shows that the 1:1 balance of the counterion exchange is violated (as long as 1 $\mathrm{MB}^{+}$displaces $1.5 \mathrm{Na}^{+}$). Such a charge imbalance is supported by the adsorption imbalance, i.e., inequality between the amount of adsorption sites, $Q_{\text {tot }}=0.57 \pm 0.03 \mathrm{mmol} / \mathrm{g}$ and the maximum adsorption capacity $Q_{\max }=0.37 \pm 0.01 \mathrm{mmol} / \mathrm{g}$, with the ratio $Q_{\text {tot }} / Q_{\max }=1.55 \pm 0.12$. The adsorption imbalance can be quite easily understood from simple steric considerations. From the geometrical point of view, at the adsorption plateau, one $\mathrm{MB}$ molecule occupies an area equal to $S_{\mathrm{MB}}=S /\left(Q_{\max } N_{\mathrm{A}}\right) \approx 0.54-0.69 \mathrm{~nm}^{2}$. The evaluated value of $S_{\mathrm{MB}}$ is consistent with the dense layer of adsorbed $\mathrm{MB}$ molecules oriented toward the surface by their C-face (Figure $S 1 b, c)$ having an area ${ }^{27} S_{\mathrm{C}} \approx 0.55-0.70 \mathrm{~nm}^{2}$. Such an orientation with the plains of the aromatic rings perpendicular to the adsorbent surface is schematically presented in Figure 6 and has been reported for $\mathrm{MB} \mathrm{H}$-aggregates adsorbed on anionic dendrimers. ${ }^{30}$

Thus, the packing density $Z_{\max }=1.6 \pm 0.2 \mathrm{~nm}^{-2}$ of adsorbed $\mathrm{MB}$ molecules at the adsorption plateau appears to be smaller than the surface density of the available adsorption sites $\left(\left|Z_{\text {tot }}\right|\right.$ $=2.5 \pm 0.3 \mathrm{~nm}^{-2}$ for SC samples or $2.2 \pm 0.4 \mathrm{~nm}^{-2}$ for SD samples). In the same vein, the hydrated sodium ions of diameter $\sim 0.5 \mathrm{~nm}$ do not have enough place at the IONP surface when it is fully covered by the MB adsorbed layer, so they are expected to be fully expelled from the IONP surface by the steric hindrance effect. This partially explains the charge imbalance at the counterion exchange. However, at the observed stoichiometry $1 \mathrm{MB}^{+}$vs $1.5 \mathrm{Na}^{+}$, we expect the effective charge density at full surface coverage by $\mathrm{MB}(\theta=1)$ to be equal to $\left|Z_{\text {eff }}\right|=\left(Q_{\text {tot }}-Q_{\max }\right) N_{\mathrm{A}} / S \sim 1 \mathrm{~nm}^{-2}$. This would imply extremely high electrophoretic mobility ( $\zeta$-potential $\sim$ $-300 \mathrm{mV})$. This is in stark contradiction with experimental values $\left|Z_{\text {eff }}\right| \ll 0.1 \mathrm{~nm}^{-2}$ at $\theta \sim 1$ displayed on Figure 5a. The effective charge remains small, and the charge imbalance has to be somehow compensated. There could exist different mechanisms of such a compensation. In general, the $\mathrm{MB}$ adsorption could involve changes in adsorption equilibria of citrate ions or protons. First, partial displacement of citrate ions by $\mathrm{MB}$ molecules could decrease the number of negatively charged carboxylate groups on the IONP surface. However, by $\mathrm{HCl}$ titration of trisodium citrate, we checked that the amount of free citrate ions in the solvent does not change with $\mathrm{MB}$ adsorption; thus, we can exclude possible citrate desorption. Second, some of the free carboxylate $\left(\mathrm{COO}^{-}\right)$groups of the bound citrate ions could progressively adsorb onto the iron oxide surface as the $\mathrm{MB}$ molecules invade the nanoparticle surface. This would decrease the structural charge $\left|Z_{\text {tot }}\right|$ and 
compensate the charge difference between $\mathrm{MB}$ and $\mathrm{Na}$ ions. Nevertheless, according to Kallay and Matijevic, ${ }^{24}$ a triple bond of the citrate ion to the iron oxide surface would require a much larger surface area per adsorbed citrate than a double bond, which is likely incompatible with the high surface density of the bound citrate, $Q_{\text {cit }}=0.55 \mathrm{mmol} / \mathrm{g}$ and $Z_{\text {cit }}=2.1$ $\mathrm{nm}^{-2}$, found in our experiments. Third, energy minimum arguments can in principle significantly displace the protonation equilibrium as a function of surface charge and the presence of adsorbed or condensed counterions. ${ }^{49}$ It is therefore possible that $\mathrm{MB}$ adsorption and repulsion of $\mathrm{Na}$ from the surface makes the protons adsorb to some of the free carboxylate groups compensating the charge difference between $\mathrm{MB}$ and $\mathrm{Na}$ ions. A weak $\mathrm{pH}$ decrease upon $\mathrm{MB}$ adsorption onto citrate-coated IONP was systematically observed in adsorption experiments without $\mathrm{pH}$ adjustment (from $\mathrm{pH}=7.7$ at $\theta=0$ to $\mathrm{pH}=6.8$ at $\theta=94 \%$ for SC samples). However, without knowledge of the $\mathrm{p} K_{\mathrm{a}}$ of the free carboxylate groups of the bound citrates, we cannot state if the observed $\mathrm{pH}$ variation is adequate to support this scenario. The second and third hypotheses are schematized in Figure 6, and they need a deeper inspection.

The third question concerning the adsorption-induced agglomeration of IONP is closely related to MB adsorption mechanisms that have to be well understood before going to agglomeration. Analysis of the MB absorption UV-visible spectra (see Figure S12 with its detailed description in the SI, Section E) allows us to conclude the following. (1) In the studied concentration range, $C_{0}=0.0141-0.0395 \mathrm{mmol} / \mathrm{L}$, $\mathrm{MB}$ is present in a monomer phase in the suspending liquid and in the $\mathrm{H}$-aggregate phase on the IONP surface, where MB molecules are laterally stacked to each other due to aromatic $\pi$ stacking interactions enhanced due to the relatively high $\mathrm{MB}$ local concentration at the surface, as reported for $\mathrm{MB}$ adsorption on polyelectrolytes, ${ }^{30-32}$ charged clays, ${ }^{50,51}$ or titanate nanowires. ${ }^{52} \mathrm{~A}$ chemical structure of the $\mathrm{H}$-aggregate is presented in Figure S1c. (2) The MB interaction with the charged IONP surface seems to be mainly electrostatic because upon addition of $0.5 \mathrm{M}$ of the sodium chloride $(\mathrm{NaCl})$ salt, the interaction seems to be screened, resulting in desorption of the major amount of $\mathrm{MB}$ and disappearance of $\mathrm{H}$-aggregates on the $\mathrm{MB}$ surface- a similar effect has been reported for $\mathrm{MB}$ adsorption on polyelectrolytes ${ }^{31,32}$ and $\mathrm{MO}$ adsorption on iron oxide/chitosan beads. ${ }^{39}$ The remaining minor part of nondesorbed $\mathrm{MB}$ upon $\mathrm{NaCl}$ addition could stem from either a change of the adsorption equilibrium in the presence of a large amount of dissolved salt or from nonelectrostatic interactions like hydrogen bonds. Unfortunately, we were unable to detect any intermolecular bond between adsorbed $\mathrm{MB}$ and citratecoated IONP by FTIR spectroscopy because of the abundant signal from iron oxide and/or insufficient spectral resolution (cf. Figure S5b and SI, Section A for details). Notice that incomplete $\mathrm{MB}$ desorption by salt addition correlates with incomplete desorption by the $\mathrm{pH}$ change, as it stems from the adsorption-desorption cycles (Figure 3).

On the basis of the described counterion condensation and $\mathrm{MB}$ adsorption behavior, we can finally propose different mechanisms of the primary agglomeration of IONP resulting from $\mathrm{MB}$ adsorption. We must however warrant the reader that we were not able to provide direct experimental proof for the below-considered mechanisms, and they have to be carefully analyzed in future.
The first possible reason for agglomeration comes from electrostatic attractive interactions due to surface domain correlations, beyond the classical DLVO model, as described in the seminal book of Evans and Wennerström. ${ }^{45}$ In fact, by analogy with adsorption of charged surfactant micellar aggregates onto an oppositely charged solid surface, one could expect a heterogeneous adsorption of $\mathrm{MB}$ molecules forming $\mathrm{H}$-aggregates on the IONP surface, as shown schematically in Figure 6. This creates a nonuniform surface charge distribution, and two approaching nanoparticles will try to accommodate a position in which low charge surface domains of one particle are faced toward high charge domains of another particle. This could considerably lower the electrostatic repulsion between particles as compared to the case of uniform charge distribution at the same average surface charge density. If some domains become positively charged, the overall electrostatic interaction can become attractive. The charge heterogeneity affects interparticle interaction on the length scale equal to the size of adsorbed $\mathrm{MB} \mathrm{H}$-aggregates. Taking the largest dimension of the $\mathrm{MB}$ molecule ${ }^{27}(R \approx 1.7$ $\mathrm{nm})$, we see that the characteristic length scale of the surface domain correlation is approximately the same as the Debye screening length $\kappa^{-1} \approx 1.5-2 \mathrm{~nm}$ for the SC-MB samples (Table 3 and Figure S8a). Thus, together with a decrease of steric repulsion (because of masking of citrate ions by adsorbed MB molecules), the surface domain correlation could reduce the repulsive barrier between nanoparticles and promote their agglomeration under van der Waals forces.

The second possible reason for agglomeration comes from attractive $\pi$-stacking interactions between two MB molecules (or two H-aggregates), each "belonging" to opposite IONP surfaces, and thus "bridging" both particles. Such a mechanism has been claimed to significantly contribute to the selfassembly of macroions by multivalent aromatic dye counterions, ${ }^{53,54}$ although electrostatic complexation was also important for multivalent dyes, as opposed to our case of monovalent $\mathrm{MB}$ dye. Alternatively, one $\mathrm{H}$-aggregate can bind to both charged surfaces thanks to the equivalence of both of their C-faces bound to two opposite particles (Figure S1b,c). However, we have to keep in mind that $\pi$-stacking interactions are very short-ranged (as opposed to surface domain correlation) and possibly cannot promote efficient selfassembly in the beginning of aggregation. It is possible that the surface domain correlation mechanism dominates at small surface coverage by $\mathrm{MB}$ and/or in the beginning of agglomeration when heterogeneous surface charge distribution is expected. At high surface coverage close to the surface packing limit of $\mathrm{MB}$, the charge should no longer be heterogeneous and short-ranged $\pi$-stacking interactions could dominate. In any case, it is very likely that $\mathrm{MB}$ adsorption and particle agglomeration are interconnected processes occurring at similar time scales. A more detailed analysis employing numerical simulations is required to shed more light into this problem.

\section{CONCLUSIONS}

This series of two papers is devoted to elucidation of the effect of molecular adsorption on the surface of magnetic nanoparticles on the enhancement of their field-induced (secondary) agglomeration and magnetic separation due to screening of the repulsive interactions between nanoparticles that involves their primary agglomeration in the absence of magnetic fields. This effect has been studied in detail for a 
particular case of charged organic dyes adsorbing onto oppositely charged iron oxide nanoparticles (IONPs). The present paper (Part I) is focused on the physicochemical mechanisms of dye adsorption and on adsorption-induced primary agglomeration of IONPs.

To this purpose, either bare or citrate-coated IONPs (maghemite $\gamma-\mathrm{Fe}_{2} \mathrm{O}_{3}$ ) have been synthesized by the coprecipitation method and their aqueous dispersions have been carefully characterized. Adsorption of cationic (methylene blue, MB) or anionic (methyl orange, MO) dyes to oppositely charged IONPs is found to be mostly promoted by relatively weak electrostatic interactions and is very sensitive to $\mathrm{pH}$ variations changing the particle surface charge and to ionic strength variations tuning the interaction length scale. However, adsorption is not fully reversible versus the $\mathrm{pH}$ or ionic strength change likely because of a weak contribution of nonelectrostatic interactions. Furthermore, the maximum surface density $Z_{\max }$ of adsorbed $\mathrm{MB}$ seems to correspond to the packing density of an adsorbed monolayer, which, depending on the molecular orientation, can be smaller than (for the MB/citrated IONP pair) or equal to (for the MB/bare IONP pair) the surface density $\left|Z_{\text {tot }}\right|$ of the available adsorption sites. Inequality between $Z_{\max }$ and $\left|Z_{\text {tot }}\right|$ seems to violate basic hypotheses of the Langmuir adsorption theory. Nevertheless, the experimental adsorption isotherms are still correctly fitted to the Langmuir law, as long as in most cases of monolayer adsorption, the isotherms have a shape similar to that of the Langmuir one. ${ }^{55}$

For the particular case of the $\mathrm{MB} /$ citrated IONP pair, $\mathrm{MB}$ is shown to form $\mathrm{H}$-aggregates on the IONP surface. The effective electric charge on the IONP surface remains nearly constant in a broad range of surface coverage by $\mathrm{MB}, 0 \leq \theta<$ $60 \%$, which is explained by the combined action of sodium counterion condensation and counterion exchange between sodium and MB. In the classical DLVO limit, the electric double-layer repulsion between IONPs seems to be unaltered by MB adsorption. Primary agglomeration of IONPs (revealed by an exponential increase of the hydrodynamic size $d_{\mathrm{H}}$ with surface coverage $\theta$ ) probably comes from surface domain correlations (as a result of heterogeneous surface charge distribution on the scale of $\mathrm{MB} \mathrm{H}$-aggregates) or $\pi$-stacking aromatic interactions between adsorbed MB molecules or $\mathrm{H}$ aggregates.

From the application perspective, the optimal adsorption conditions have been determined for the two dyes. The maximum adsorption capacity of $\mathrm{MB}$ is equal to $139 \pm 4 \mathrm{mg} / \mathrm{g}$, and it is obtained with citrate-coated nanoparticles in a large $\mathrm{pH}$ range (from 4 to 11 ). On the other hand, the maximum adsorption capacity of $\mathrm{MO}$ with bare nanoparticles is greater than for the $\mathrm{MB}$, and it is equal to $257 \pm 16 \mathrm{mg} / \mathrm{g}$ in an acid medium $(\mathrm{pH} \sim 4)$. The reusability of IONPs was checked with $\mathrm{MB}$ and citrate-coated nanoparticles. The adsorption efficiency remains about $99 \%$ even after nine adsorption/desorption cycles of the dye. We can conclude that, besides its fundamental interest, this study could also be helpful in the development of magnetic adsorbents; IONPs present a high application potential for the recovery of charged pollutants from water due to their magnetic properties, adsorption efficiency, and reusability. A further step to the practical application would be development of the magnetic composites allowing simultaneous removal of cationic and anionic dyes. This would be possible using carbon materials (like graphene oxide), having a strong affinity to both charged species. $^{56}$
Another strategy consists of using specially designed IONPs for catalytic degradation of dyes ${ }^{57}$ the technique not requiring dye adsorption but necessitating recovery of catalytic properties of the IONP surface for multicycle application.

Based on the present results, the companion paper (Part II) will report the enhanced magnetic separation of IONPs thanks to primary agglomeration of nanoparticles due to dye adsorption on their surface.

\section{EXPERIMENTAL SECTION}

4.1. Chemicals. Methylene blue (MB), methyl orange (MO), and trisodium citrate were supplied in a powder form by Sigma-Aldrich and used without any prior purification. Chemical formula, molecular weight, molecular structure, and $\mathrm{p} K_{\mathrm{a}}$ of $\mathrm{MB}, \mathrm{MO}$, and trisodium citrate are shown in the SI (Figure S1 and Table S1). Stock solutions of dyes were obtained by dissolving the powder in Milli-Q water followed by an Orbital shaker stirring for $30 \mathrm{~min}$. All other reagents and solvents were purchased and used without further purification.

4.2. Preparation of IONPs. IONPs were synthesized according to Massart's method ${ }^{17,18}$ by alkaline coprecipitation in concentrated ammonium hydroxide of an aqueous stoichiometric mixture of $\mathrm{FeCl}_{2}$ and $\mathrm{FeCl}_{3}$. The resulting dark-black magnetite $\left(\mathrm{Fe}_{3} \mathrm{O}_{4}\right)$ was then stirred in nitric acid, oxidized into maghemite $\left(\gamma-\mathrm{Fe}_{2} \mathrm{O}_{3}\right)$ by a boiling solution of $\mathrm{Fe}\left(\mathrm{NO}_{3}\right)_{3}$, and, after washing, dispersed into distilled water, leading to a stable suspension denoted the FFA sample. At this time, nanoparticles, positively charged with nitrate counterions, are dispersed in dilute nitric acid solution. The $\mathrm{pH}$ value of the resulting suspension is about 2 . To obtain magnetic citratecoated nanoparticles, solid trisodium citrate was added to the ferrofluid FFA, the molar ratio $C_{\mathrm{cit}} / C_{\mathrm{Fe}}$ being equal to 0.12 with $C_{\text {cit }}$ and $C_{\mathrm{Fe}}$ being the molar concentrations of added citrate ions and iron in FFA, respectively. Then, the mixture was stirred for $30 \mathrm{~min}$ at $80^{\circ} \mathrm{C}$. By adding citrate, a selfassembly between the negatively charged citrate and positively charged nanoparticles occurs, leading to the formation of a dark-brown precipitate, which was recovered from the reaction mixture using a permanent magnet. Then, IONPs were washed three times using acetone and diethyl ether and dispersed in an appropriate volume of Milli-Q water. A stable ferrofluid denoted the FFCit sample was obtained. It consists of negatively charged citrate-coated nanoparticles, dispersed in a diluted sodium citrate solution. Notice that free sodium citrate appears in the solvent because the excess was not completely removed from the solvent after IONP washing, which allows thermodynamic equilibrium between the adsorbed and free citrate ions. Free carboxylate functions of the adsorbed citrate are at the origin of the negative surface charge, and the counterions are sodium ions. The $\mathrm{pH}$ value of the citrated ferrofluid is about 7.5. The two parent ferrofluids FFA and FFCit remain stable for years.

4.3. Experimental Methods. Iron and sodium concentrations in IONP suspensions were determined by AAS using an air-acetylene flame (Perkin Elmer AAnalyst 100 apparatus). The sodium-ion concentration was also measured using ICPAES (Perkin Elmer Optima $8000 \mathrm{DV}$ ) at two wavelengths $\lambda=$ $330.237 \mathrm{~nm}$ and $589.592 \mathrm{~nm}$ after appropriate internal calibration. Before these measurements, samples with magnetic nanoparticles were heated to boiling point in concentrated hydrochloric acid $(\approx 12 \mathrm{~mol} / \mathrm{L})$ until total dissolution of the nanoparticles into iron (III) ions was achieved. From the iron molar concentration of the ferrofluid $\left(C_{\mathrm{Fe}}\right)$, the weight 
concentration of iron oxide $\left(c_{\mathrm{w}}\right)$ and the magnetic nanoparticle volume fraction $(\varphi)$ were deduced, $\varphi=c_{\mathrm{w}} / \rho$, with $\rho=5.074$ $\mathrm{g} / \mathrm{cm}^{3}$ being the maghemite density. FTIR spectra of trisodium citrate, MB, FFA, FFCit, and MB/FFCit samples were recorded in $\mathrm{KBr}$ discs in the wavenumber range of 4000$400 \mathrm{~cm}^{-1}$ on a Bruker Tensor 27 apparatus. In the cases of FFCit and MB/FFCit samples, the nanoparticles were precipitated, and the supernatant was rapidly eliminated to minimize the quantity of free citrates and/or MB molecules before the FTIR analysis. The amount of citrate adsorbed on nanoparticles was estimated from TGA performed on dried nanoparticles in a $\mathrm{N}_{2}$ atmosphere at a heating rate of $10{ }^{\circ} \mathrm{C} /$ min (TA Instruments SDT Q600). The morphology of the magnetic nanoparticles was observed by TEM using a Jeol model JEM $100 \mathrm{CX}$ at $100 \mathrm{kV}$. The magnetization curves of parent ferrofluids were measured at room temperature using a homemade vibrating sample magnetometer (VSM) based on the one described in ref 58. The magnetic size distribution of the nanoparticles, which can be approximated by a log-normal law, was obtained by fitting magnetization curve parameters and led to the median diameter $d_{\mathrm{M}}\left(\ln d_{\mathrm{M}}=\langle\ln d\rangle\right)$ and distribution width $\sigma_{\mathrm{M}}$ of the nanoparticles. ${ }^{59} \mathrm{XRD}$ on dried samples using a Rigaku Ultima IV apparatus with a copper anode $(\mathrm{Cu} \mathrm{K} \alpha$ radiation, $\lambda=1.54178 \AA$ ) was performed to identify the crystallographic structure.

The structural surface charge of the bare nanoparticles (FFA sample) was obtained by potentiometric titration and conductivity measurements performed on FFA following the methodology of Campos et al. ${ }^{60}$ The surface charge density $\left(Z_{\text {tot }}<0\right)$ of the citrate-coated (FFCit sample) nanoparticles (i.e., the number of elementary charges $e$ per unit surface in $\mathrm{nm}^{-2}$, including only negative charges of carboxylate groups) was estimated from the quantity of sodium counterions of the carboxylate groups ( $Q_{\mathrm{Na}}$ in mmol per $\mathrm{g}$ of iron oxide), $Z_{\text {tot }}=$ $-Q_{\mathrm{Na}_{\mathrm{A}}} N_{\mathrm{A}} / S$, where $N_{\mathrm{A}} \approx 6.0 \times 10^{23} \mathrm{~mol}^{-1}$ is the Avogadro number, $S$ is the geometric specific area of citrated IONP, estimated by modeling each particle as a sphere with a diameter $d, S \approx 6 /(\rho d)$, and $Q_{\mathrm{Na}}=\left(C_{\mathrm{Na}}{ }^{0}-C_{\mathrm{Na}}\right) / c_{\mathrm{w}}$, with $C_{\mathrm{Na}}{ }^{0}$ $(\mathrm{mmol} / \mathrm{L})$ being the total sodium concentration of the ferrofluid sample and $C_{\mathrm{Na}}(\mathrm{mmol} / \mathrm{L})$ being the sodium concentration in the supernatant obtained after the removal of IONP. To check the agglomeration state of IONP, hydrodynamic size measurements were performed by DLS using Zetasizer NanoZS (Malvern Instruments, U.K.) operating at a $173^{\circ}$ scattering angle. The $Z$-average hydrodynamic diameter, $d_{\mathrm{H}}$, and the PDI were obtained from a fit of the measured DLS correlogram by the second-order Cumulant method, while the size distribution over the intensity of the diffused light (intensity distribution) was obtained from a multiexponent fit using the standard CONTIN fit. Electrophoretic mobility measurements were realized using the same Zetasizer apparatus on FFCit and MB/FFCit samples, while $\mathrm{pH}$ and electric conductivity were also measured on the supernatant of these samples. The effective charge density $Z_{\text {eff }}$ $<0$ (i.e., the surface density of the structural charge, in $\mathrm{nm}^{-2}$, partially screened by adsorbed or condensed counterions) was evaluated from the measured electrophoretic mobility $\mu_{\mathrm{E}}$ of the porous IONP agglomerates and the Debye screening length $\kappa^{-1}$, this last being evaluated through both conductivity measurements and sodium-ion concentration measurements (see details in the SI; Sections B and C).

During dye adsorption experiments, the dye concentration was measured after appropriate calibration, by spectropho- tometry using a UV-visible UVIKON XL Secoman apparatus at a fixed wavelength $\lambda=664 \mathrm{~nm}(\mathrm{MB})$ and $\lambda=460 \mathrm{~nm}$ (MO).

4.4. Adsorption/Desorption Experiments. Batch adsorption experiments were carried out at room temperature $\left(\approx 20{ }^{\circ} \mathrm{C}\right)$. The dye-IONP samples (with the dye standing for $\mathrm{MB}$ or MO) were prepared as follows. An appropriate amount of the dye stock solution was added to the diluted ferrofluid to obtain dye-IONP samples with the desired initial concentration of dye $C_{0}$; the total volume is equal to $10 \mathrm{~mL}$, and the weight concentration of iron oxide $\left(c_{\mathrm{w}}\right)$ is about $2 \mathrm{~g} / \mathrm{L}$. If necessary, the $\mathrm{pH}$ of the samples was adjusted using either $\mathrm{HNO}_{3}$ or $\mathrm{NaOH}$ solutions. The samples were stirred using an Orbital shaker (IKA KS 260) for the desired time ranging between 25 and $350 \mathrm{~min}$. Magnetic nanoparticles with the adsorbed dye were then extracted from solution, either using a permanent magnet when the sample is flocculated or by centrifugation on a Macrosep Advance centrifugal filter (VWR, France, $30 \mathrm{kDa}$ ) for stable samples. The molar concentration $(\mathrm{mmol} / \mathrm{L})$ of dye remaining in the solution (nonadsorbed molecules) at equilibrium $\left(C_{\mathrm{eq}}\right)$ or at time $t\left(C_{t}\right)$ was measured by UV-visible spectrophotometry, as described in Section 4.3. The amount of adsorbed dye at equilibrium $\left(Q_{\mathrm{eq}}\right)$ or at time $t$ $\left(Q_{t}\right)$, expressed in $\mathrm{mmol} / \mathrm{g}$ of iron oxide, was deduced using the mass balance equation $Q_{t}=\left(C_{0}-C_{t}\right) / c_{\mathrm{w}}$. Based on these measurements, experimental dependences of the adsorbed dye concentration at equilibrium $Q_{\mathrm{eq}}$ versus the molar concentration of free dye $C_{\mathrm{eq}}$, called the adsorption isotherm, were plotted. In addition to them, kinetics of dye adsorption and effect of $\mathrm{pH}$ on adsorption were also investigated for $C_{0} \approx 0.4$ $\mathrm{mmol} / \mathrm{L}$ and $c_{\mathrm{w}} \approx 2 \mathrm{~g} / \mathrm{L}$, and the obtained results were analyzed in terms of the adsorption efficiency, defined as the ratio of the amount of adsorbed dye to the initial concentration of the introduced dye: $E(t)=Q_{t} c_{\mathrm{w}} / C_{0}$ for kinetic studies or $E_{\mathrm{eq}}$ $=Q_{\mathrm{eq}} c_{\mathrm{w}} / C_{0}$ for $\mathrm{pH}$-effect studies.

In some cases, for the citrated $\mathrm{MB} / \mathrm{IONP}$ pair, we analyzed the full UV-visible adsorption spectra of either the supernatant free of nanoparticles or MB/IONP samples to check the presence of $\mathrm{MB}$ molecular aggregates. In addition, citrated $\mathrm{MB} / \mathrm{IONP}$ adsorption experiments were accompanied by measurements of sodium and citrate ion concentrations in the supernatant to check the counterion exchange induced by $\mathrm{MB}$ adsorption and possible displacement of adsorbed citrate ions by adsorbing $\mathrm{MB}$ molecules. Sodium was detected by ICP-AES as described above, while free citrate was determined by $\mathrm{HCl}$ acid titration of the trisodium citrate base using a Mettler Toledo G20S automatic titrator.

To investigate the reusability of the IONP, adsorption/ desorption cycles were realized with $\mathrm{MB} / \mathrm{FFCit}$ samples. An appropriate amount of FFCit was first added to $10 \mathrm{~mL}$ of an MB solution $\left(C_{0} \approx 4 \mathrm{mmol} / \mathrm{L}, c_{\mathrm{w}} \approx 20 \mathrm{~g} / \mathrm{L}\right)$ at $\mathrm{pH} 8$, and the solution was stirred for $1 \mathrm{~h}$. Then, the nanoparticles were magnetically recovered, gently washed with distilled water, and introduced for $30 \mathrm{~min}$ into nitric acid solution at a $\mathrm{pH}$ value close to 2 . Then, the particles were again separated from the solution. The quantity of desorbed dye in the supernatant was determined by UV-visible spectrophotometry. Finally, IONPs were washed with distilled water, sodium hydroxide was added to raise the $\mathrm{pH}$ to 8 , and nine adsorption/desorption cycles were conducted. 


\section{ASSOCIATED CONTENT}

\section{(3) Supporting Information}

The Supporting Information is available free of charge at https://pubs.acs.org/doi/10.1021/acsomega.1c02401.

Physicochemical characterizations (Section A); evaluation of the Debye screening length (section B); evaluation of the effective surface charge (Section $\mathrm{C}$ ); adsorption of dyes on IONP (Section D); analysis of the UV-visible absorption spectra of MB in FFCit samples (Section E) (PDF)

\section{AUTHOR INFORMATION}

\section{Corresponding Author}

Agnès Bee - Sorbonne Université, CNRS, UMR 8234, PHENIX, 75252 Paris Cedex 5, France; Email: agnes.bee@ sorbonne-universite.fr

\section{Authors}

Delphine Talbot - Sorbonne Université, CNRS, UMR 8234, PHENIX, 75252 Paris Cedex 5, France

Jordy Queiros Campos - Université Côte d'Azur, CNRS UMR 7010 Institute of Physics of Nice (INPHYNI), 06108 Nice, France

Blanca L. Checa-Fernandez - Department of Applied Physics, University of Granada, 18071 Granada, Spain; CEITBasque Research and Technology Alliance (BRTA) and Tecnun, University of Navarra, 20018 Donostia/San Sebastián, Spain

Jéssica A. Marins - Université Côte d'Azur, CNRS UMR 7010 Institute of Physics of Nice (INPHYNI), 06108 Nice, France; 10 orcid.org/0000-0002-3972-3957

Claire Lomenech - Université Côte d'Azur, CNRS UMR 7010 Institute of Physics of Nice (INPHYNI), 06108 Nice, France

Charlotte Hurel - Université Côte d'Azur, CNRS UMR 7010 Institute of Physics of Nice (INPHYNI), 06108 Nice, France

Guilhem D. Godeau - Université Côte d'Azur, CNRS UMR 7010 Institute of Physics of Nice (INPHYNI), 06108 Nice, France

Maxime Raboisson-Michel - Université Côte d'Azur, CNRS UMR 7010 Institute of Physics of Nice (INPHYNI), 06108 Nice, France; Axlepios Biomedical, 06510 Carros, France

Gregory Verger-Dubois - Axlepios Biomedical, 06510 Carros, France

Layaly Obeid - Sorbonne Université, CNRS, UMR 8234, PHENIX, 75252 Paris Cedex 5, France

Pavel Kuzhir - Université Côte d'Azur, CNRS UMR 7010 Institute of Physics of Nice (INPHYNI), 06108 Nice, France; (1) orcid.org/0000-0001-7089-6197

Complete contact information is available at:

https://pubs.acs.org/10.1021/acsomega.1c02401

\section{Notes}

The authors declare no competing financial interest.

\section{ACKNOWLEDGMENTS}

The authors are very grateful to Thomas Ducasse for his experimental assistance and to Pr. Jacques Persello for helpful discussions. P.K., C.L., C.H., and G.D.G. acknowledge the French "Agence Nationale de la Recherche", Project Future Investments UCA JEDI, No. ANR-15-IDEX-01 (projects MANTRA, ImmunoMag, and MagFilter) and the private company Axlepios Biomedical for financial support. J.Q.C. acknowledges the financial support of UCA JEDI and Axlepios Biomedical through the Ph.D. fellowship. BLCF acknowledges Erasmus Mundus through its mobility fellowship.

\section{REFERENCES}

(1) Yavuz, C. T.; Mayo, J. T.; Yu, W. W.; et al. Low field Magnetic separation of monodisperse Fe3O4 monocrystals. Science 2006, 314, 964-967.

(2) Ambashta, R. D.; Sillanpää, M. Water purification using magnetic assistance. J. Hazard. Mater. 2010, 180, 38-49.

(3) Gijs, M. A.; Lacharme, F.; Lehmann, U. Microfluidic applications of magnetic particles for biological analysis and catalysis. Chem. Rev. 2010, 110, 1518-1563.

(4) Douziech-Eyrolles, L.; Marchais, H.; Herve, K.; Munnier, E.; Souce, M.; Linassier, C.; Dubois, P.; Chourpa, I. Nanovectors for anticancer agents based on superparamagnetic iron oxide nanoparticles. Int. J. Nanomed. 2007, 2, 541-550.

(5) Hergt, R.; Dutz, S.; Müller, R.; Zeisberger, M. Magnetic particle hyperthermia: nanoparticle magnetism and materials development for cancer therapy. J. Phys.: Condens. Matter 2006, 18, S2919.

(6) Bouchlaka, M. N.; Sckisel, G. D.; Wilkins, D.; Maverakis, E.; Monjazeb, A. M.; Fung, M.; Welniak, L.; Redelman, D.; Fuchs, A.; Evrensel, C. A.; Murphy, W. J. Mechanical disruption of tumors by iron particles and magnetic field application results in increased antitumor immune responses. PLoS ONE 2012, 7, No. e48049.

(7) Okoli, C.; Fornara, A.; Qin, J.; Toprak, M. S.; Dalhammar, G.; Muhammed, M.; Rajarao, G. K. Characterization of superparamagnetic iron oxide nanoparticles and its application in protein purification. J. Nanosci. Nanotechnol. 2011, 11, 10201-10206.

(8) Scherer, F.; Anton, M.; Schillinger, U.; Henke, J.; Bergemann, C.; Krüger, A.; Gansbacher, B.; Plank, C. Magnetofection: enhancing and targeting gene delivery by magnetic force in vitro and in vivo. Gene Ther. 2002, 9, 102.

(9) Guibert, C.; Dupuis, V.; Peyre, V.; Fresnais, J. Hyperthermia of magnetic nanoparticles: experimental study of the role of aggregation. J. Phys. Chem. C 2015, 119, 28148-28154.

(10) Etheridge, M. L.; Hurley, K. R.; Zhang, J.; Jeon, S.; Ring, H. L.; Hogan, C.; Haynes, ChL.; Garwood, M.; Bischof, J. C. Accounting for biological aggregation in heating and imaging of magnetic nanoparticles. Technology 2014, 02, 214-228.

(11) Eberbeck, D.; Kettering, M.; Bergemann, C.; Zirpel, P.; Hilger, I.; Trahms, L. Quantification of the aggregation of magnetic nanoparticles with different polymeric coatings in cell culture medium. J. Phys. D: Appl. Phys. 2010, 43, No. 405002.

(12) Cabrera, D.; Camarero, J.; Ortega, D.; Teran, F. J. Influence of the aggregation, concentration, and viscosity on the nanomagnetism of iron oxide nanoparticle colloids for magnetic hyperthermia. J. Nanopart. Res. 2015, 17, No. 121.

(13) de las Cuevas, G.; Faraudo, J.; Camacho, J. Low-gradient magnetophoresis through field-induced reversible aggregation. J. Phys. Chem C 2008, 112, 945-950.

(14) Leong, S. S.; Ahmad, Z.; Low, S. C.; Camacho, J.; Faraudo, J.; Lim, J. Unified view of magnetic nanoparticle separation under magnetophoresis. Langmuir 2020, 36, 8033-8055.

(15) Kuzhir, P.; Magnet, C.; Ezzaier, H.; Zubarev, A.; Bossis, G. Magnetic filtration of phase separating ferrofluids: From basic concepts to microfluidic device. J. Magn. Magn. Mater. 2017, 431, 84-90.

(16) Ezzaier, H.; Marins, J. A.; Claudet, C.; Hemery, G.; Sandre, O.; Kuzhir, P. Kinetics of aggregation and magnetic separation of multicore iron oxide nanoparticles: effect of the grafted layer thickness. Nanomaterials 2018, 8, 623.

(17) Massart, R. Preparation of aqueous magnetic liquids in alkaline and acidic media. IEEE Trans. Magn. 1981, 17, 1247-1248.

(18) Fauconnier, N.; Bee, A.; Roger, J.; Pons, J. N. Synthesis of aqueous magnetic liquids by surface complexation of maghemite nanoparticles. J. Mol. Liq. 1999, 83, 233-242. 
(19) Bee, A.; Massart, R.; Neveu, S. Synthesis of very fine maghemite particles. J. Magn. Magn. Mater. 1995, 149, 6-9.

(20) Fresnais, J.; Berret, J. F.; Qi, L.; Chapel, J. P.; Castaing, J. C.; Sandre, O.; Frka-Petesic, B.; Perzynski, R.; Oberdisse, J.; Cousin, F. Universal scattering behavior of coassembled nanoparticle-polymer clusters. Phys. Rev. E 2008, 78, No. 040401.

(21) Zhang, Y.; Kallay, N.; Matijevic, E. Interaction of metal hydrous oxides with chelating agents. 7. Hematite-oxalic acid and-citric acid systems. Langmuir 1985, 1, 201-206.

(22) Cornell, R. M.; Schindler, P. W. Infrared study of the adsorption of hydroxycarboxylic acids on $\alpha$-FeOOH and amorphous Fe (III) hydroxide. Colloid Polym. Sci. 1980, 258, 1171-1175.

(23) Hidber, P. C.; Graule, T. J.; Gauckler, L. J. Citric acid-a dispersant for aqueous alumina suspensions. J. Am. Ceramic Soc. 1996, 79, 1857-1867.

(24) Kallay, N.; Matijevic, E. Adsorption at solid/solution interfaces. 1. Interpretation of surface complexation of oxalic and citric acids with hematite. Langmuir 1985, 1, 195-201.

(25) Dubois, E.; Cabuil, V.; Boué, F.; Perzynski, R. Structural analogy between aqueous and oily magnetic fluids. J. Chem. Phys. 1999, 111, 7147-7160.

(26) Hill, T. L. An Introduction to Statistical Thermodynamics; Dover Publications Inc.: New York, 1986.

(27) Imamura, K.; Ikeda, E.; Nagayasu, T.; Sakiyama, T.; Nakanishi, $\mathrm{K}$. Adsorption behavior of methylene blue and its congeners on a stainless steel surface. J. Colloid Interface Sci. 2002, 245, 50-57.

(28) Huang, J. H.; Huang, K. L.; Liu, S. Q.; Wang, A. T.; Yan, C. Adsorption of Rhodamine $\mathrm{B}$ and methyl orange on a hypercrosslinked polymeric adsorbent in aqueous solution. Colloids Surf., A 2008, 330, $55-61$.

(29) Zhang, Y.; Sun, B.; Deng, S.; Wang, Y.; Peng, H.; Li, Y.; Zhang, $X$. Methyl orange degradation by pulsed discharge in the presence of activated carbon fibers. Chem. Eng. J. 2010, 159, 47-52.

(30) Jockusch, S.; Turro, N. J.; Tomalia, D. A. Aggregation of methylene blue adsorbed on starburst dendrimers. Macromolecules 1995, 28, 7416-7418.

(31) Moreno-Villoslada, I.; Torres, C.; González, F.; Shibue, T.; Nishide, $\mathrm{H}$. Binding of methylene blue to polyelectrolytes containing sulfonate groups. Macromol. Chem. Phys. 2009, 210, 1167-1175.

(32) Moreno-Villoslada, I.; Torres-Gallegos, C.; Araya-Hermosilla, R.; Nishide, H. Influence of the linear aromatic density on methylene blue aggregation around polyanions containing sulfonate groups. $J$. Phys. Chem. B 2010, 114, 4151-4158.

(33) Goodman, S. M.; Bura, R.; Dichiara, A. B. Facile impregnation of graphene into porous wood filters for the dynamic removal and recovery of dyes from aqueous solutions. ACS Appl. Nano Mater. 2018, 1, 5682-5690.

(34) Tan, K. B.; Vakili, M.; Horri, B. A.; Poh, P. E.; Abdullah, A. Z.; Salamatinia, B. Adsorption of dyes by nanomaterials: recent developments and adsorption mechanisms. Sep. Purif. Technol. 2015, 150, 229-242.

(35) Gupta, N.; Pant, P.; Gupta, C.; Goel, P.; Jain, A.; Anand, S.; Pundir, A. Engineered magnetic nanoparticles as efficient sorbents for wastewater treatment: a review. Mater. Res. Innovations 2017, 22, 434-450.

(36) Ji, Y.; Ma, C.; Li, J.; Zhao, H.; Chen, Q.; Li, M.; Liu, H. A magnetic adsorbent for the removal of cationic dyes from wastewater. Nanomaterials 2018, 8, 710.

(37) Li, J.; Zhao, H.; Ma, C.; Han, Q.; Li, M.; Liu, H. Preparation of Fe3O4@ polyoxometalates nanocomposites and their efficient adsorption of cationic dyes from aqueous solution. Nanomaterials 2019, 9, 649.

(38) Anushree, C.; Philip, J. (2019). Efficient removal of methylene blue dye using cellulose capped $\mathrm{Fe} 3 \mathrm{O} 4$ nanofluids prepared using oxidation-precipitation method. Colloids Surf., A 2019, 567, 193-204.

(39) Obeid, L.; Bée, A.; Talbot, D.; Jaafar, S. B.; Dupuis, V.; Abramson, S.; Cabuil, V.; Welschbillig, M. Chitosan/maghemite composite: A magsorbent for the adsorption of methyl orange. $J$. Colloid Interface Sci. 2013, 410, 52-58.
(40) Kyzas, G. Z.; Bikiaris, D. N.; Mitropoulos, A. C. Chitosan adsorbents for dye removal: a review. Polym. Int. 2017, 66, 18001811.

(41) Dubois, E.; Perzynski, R.; Boué, F.; Cabuil, V. Liquid- gas transitions in charged colloidal dispersions: small-angle neutron scattering coupled with phase diagrams of magnetic fluids. Langmuir 2000, 16, 5617-5625.

(42) Cousin, F.; Dubois, E.; Cabuil, V. Tuning the interactions of a magnetic colloidal suspension. Phys. Rev. E 2003, 68, No. 021405.

(43) Lattuada, M.; Wu, H.; Morbidelli, M. Hydrodynamic radius of fractal clusters. J. Colloid Interface Sci. 2003, 268, 96-105.

(44) Miller, N. P.; Berg, J. C.; O’Brien, R. W. The electrophoretic mobility of a porous aggregate. J. Colloid Interface Sci. 1992, 153, 237-243.

(45) Evans, D. F.; Wennerstrom, H. The Colloidal Domain - Where Physics, Chemistry, Biology, and Technology Meet, 2nd ed; Wiley-VCH: USA, 1999.

(46) Manning, G. S. Limiting laws and counterion condensation in polyelectrolyte solutions I. Colligative properties. J. Chem. Phys. 1969, 51, 924-933.

(47) Zimm, B. H.; Bret, M. L. Counter-ion condensation and system dimensionality. J. Biomol. Struct. Dyn. 1983, 1, 461-471.

(48) Manning, G. S. Counterion condensation on charged spheres, cylinders, and planes. J. Phys. Chem. B 2007, 111, 8554-8559.

(49) Gunnarsson, M.; Abbas, Z.; Ahlberg, E.; Nordholm, S. Corrected Debye-Hückel analysis of surface complexation: III. Spherical particle charging including ion condensation. J. Colloid Interface Sci. 2004, 274, 563-578.

(50) Gessner, F.; Schmitt, C. C.; Neumann, M. G. Time-dependent spectrophotometric study of the interaction of basic dyes with clays. I. Methylene blue and neutral red on montmorillonite and hectorite. Langmuir 1994, 10, 3749-3753.

(51) Neumann, M. G.; Gessner, F.; Schmitt, C. C.; Sartori, R. Influence of the layer charge and clay particle size on the interactions between the cationic dye methylene blue and clays in an aqueous suspension. J. Colloid Interface Sci. 2002, 255, 254-259.

(52) Horváth, E.; Szilágyi, I.; Forró, L.; Magrez, A. Probing titanate nanowire surface acidity through methylene blue adsorption in colloidal suspension and on thin films. J. Colloid Interface Sci. 2014, 416, 190-197.

(53) Willerich, I.; Li, Y.; Gröhn, F. (2010). Influencing Particle Size and Stability of Ionic Dendrimer- Dye Assemblies. J. Phys. Chem. B 2010, 114, 15466-15476.

(54) Mariani, G.; Moldenhauer, D.; Schweins, R.; Gröhn, F. Elucidating electrostatic self-assembly: Molecular parameters as key to thermodynamics and nanoparticle shape. J. Am. Chem. Soc. 2016, $138,1280-1293$.

(55) Patrykiejew, A.; Jaroniec, M. Partially mobile adsorption of gases on solid surfaces. Adv. Colloid Interface Sci. 1984, 20, 273-339.

(56) Yu, Z.; Hu, C.; Dichiara, A. B.; Jiang, W.; Gu, J. Cellulose nanofibril/carbon nanomaterial hybrid aerogels for adsorption removal of cationic and anionic organic dyes. Nanomaterials 2020, 10, 169.

(57) Anushree, C.; Krishna, D. N. G.; Philip, J. Efficient Dye Degradation via Catalytic Persulfate Activation using Iron OxideManganese Oxide Core-Shell Particle Doped with Transition Metal Ions. J. Mol. Liq. 2021, 337, No. 116429.

(58) Foner, S.; McNiff, E. J., Jr. Very low frequency integrating vibrating sample magnetometer (VLFVSM) with high differential sensitivity in high dc fields. Rev. Sci. Instrum. 1968, 39, 171-179.

(59) Cabuil, V.; Perzynski, R. Particle size determination in magnetic fluids. In Magnetic Fluids and Handbook Applications; Berkovski, V.; Bashtovoi, V., Eds.; Begell house: New York and Wallinford (UK), 1996.

(60) Campos, A.; Tourinho, F. A.; da Silva, G.; Lara, M. C. F. L.; Depeyrot, J. Nanoparticles superficial density of charge in electric double-layered magnetic fluid: A conductimetric and potentiometric approach. Eur. Phys. J. E 2001, 6, 29-35. 